\title{
ON THE HIGH-ORDER COEFFICIENTS IN THE UNIFORM ASYMPTOTIC EXPANSION FOR THE INCOMPLETE GAMMA FUNCTION
}

\author{
T. M. Dunster, R. B. Paris, and S. Cang
}

\begin{abstract}
We examine the asymptotic nature as $k \rightarrow \infty$ of the coefficients $c_{k}(\eta)$ appearing in the uniform asymptotic expansion of the incomplete gamma function $\Gamma(a, z)$ where $\eta$ is a variable that depends on the ratio $z / a$. It is shown that this expansion diverges like the familiar "factorial divided by a power" dependence multiplied by a function $f_{k}(\eta)$. For values of $\eta$ near the real axis, $f_{k}(\eta)$ is a slowly varying function, but in the left half-plane, there are two lobes situated symmetrically about the negative real $\eta$ axis in which $f_{k}(\eta)$ becomes large. The asymptotic expansion of $f_{k}(\eta)$ as $k \rightarrow \infty$ is found to reveal a resurgence-type structure in which the high-order coefficients are related to the low-order coefficients. Numerical examples are given to illustrate the growth of the coefficients $c_{k}(\eta)$
\end{abstract}

\section{Introduction}

The incomplete gamma function $\Gamma(a, z)$ occupies a central position in the mathematical literature as one of the most fundamental and important special functions of analysis. This position recently has been reinforced in the development of the new theory of exponentially-improved asymptotic expansions and the associated new interpretation of the Stokes phenomenon where $\Gamma(a, z)$ is used as an approximant [9]. The incomplete gamma function also has been shown to have an intimate connection with the Riemann zeta function $\zeta(s)$ and to play an important role in new asymptotic methods for its accurate computation on the critical line $[10 ; 11]$. The motivation for the present study arose from this last application in the examination of the growth of the high-order coefficients in an asymptotic formula for $\zeta\left(\frac{1}{2}+i t\right)$ as $t \rightarrow \infty$ [11].

The asymptotic expansion of the normalized incomplete gamma function $Q(a, z) \equiv$ $\Gamma(a, z) / \Gamma(a)$ as $a \rightarrow \infty$ is given by Temme $[13 ; 14, \S 11.2]$ as

$$
Q(a, z) \sim \frac{1}{2} \operatorname{erfc}\left(\eta \sqrt{\frac{1}{2} a}\right)+\frac{e^{-\frac{1}{2} a \eta^{2}}}{\sqrt{2 \pi a}} \sum_{k=0}^{\infty} c_{k}(\eta) a^{-k}
$$

where

$$
\eta=\{2(\mu-\ln (1+\mu))\}^{\frac{1}{2}}
$$

and

$$
\mu=\lambda-1, \quad \lambda=z / a
$$

Received April 29, 1997, revised January 22, 1998.

1991 Mathematics Subject Classification: 30E15, 33B20.

Key words and phrases: incomplete gamma function, asymptotic expansion, resurgence. 
The branches on the right-hand side of (1.2) are chosen so that $\eta(\mu)$ is analytic in the vicinity of $\mu=0(z=a)$, with $\eta \sim \mu$ as $\mu \rightarrow 0$. The coefficients $c_{k}(\eta)$ are defined recursively by

$$
c_{0}(\eta)=\frac{1}{\mu}-\frac{1}{\eta}, \quad c_{k}(\eta)=\frac{1}{\eta} \frac{d}{d \eta} c_{k-1}(\eta)+\frac{\gamma_{k}}{\mu}, \quad(k \geq 1)
$$

and possess the general structure [13, Eq. (3.3)]

$$
c_{k}(\eta)=(-)^{k}\left\{\frac{Q_{k}(\mu)}{\mu^{2 \kappa}}-\frac{2^{k} \Gamma(\kappa)}{\Gamma\left(\frac{1}{2}\right) \eta^{2 \kappa}}\right\}, \quad \kappa=k+\frac{1}{2},
$$

in which $Q_{k}(\mu)$ is some polynomial in $\mu$ of degree $2 k$; from (1.4), the first two polynomials are $Q_{0}(\mu)=1$ and $Q_{1}(\mu)=1+\mu+\mu^{2} / 12$. The coefficients $\gamma_{k}$ are those appearing in Stirling's formula:

$$
\Gamma(z) \sim(2 \pi)^{\frac{1}{2}} z^{z-\frac{1}{2}} e^{-z} \sum_{k=0}^{\infty}(-)^{k} \gamma_{k} z^{-k}, \quad(z \rightarrow \infty, \quad|\arg (z)|<\pi) ;
$$

the first few Stirling coefficients are $\gamma_{0}=1, \gamma_{1}=-1 / 12, \gamma_{2}=1 / 288, \gamma_{3}=139 / 51840$, and $\gamma_{4}=-571 / 2488320$.

The expansion (1.1) holds uniformly in $|z| \in[0, \infty)$ for $z / a$ lying in some unbounded domain $\Xi(a)$, say, whose boundaries depend on $\arg (a)$. For the case $\arg (a)=0$, the domain is described in $\S 3$ of [6] and is slightly larger than the sheet $|\arg (z / a)| \leq \frac{3}{2} \pi$. For other values of $a$, such that $|\arg (a)| \leq \frac{1}{2} \pi$, the expansion certainly holds for $|\arg (z / a)| \leq \pi$, with extensions to other values of $\arg (z / a)$ possible via the wellknown analytic continuation formula (6.1) given below. We remark that the domain $\Xi(a)$ is not quite as large as claimed in [13]. For the case $|\arg (-a)| \leq \frac{1}{2} \pi$, one would express $\Gamma(a, z)$ in terms of the generalized exponential integral [8, Chap. 2, Ex. 5.1], and an asymptotic expansion, uniformly valid for $|z| \in[0, \infty)$ with $|\arg (z / a)| \leq \pi$, would then be obtainable from [7].

We shall focus attention on the growth of the coefficients $c_{k}(\eta)$ as $k$ becomes large. We remark that an expansion for $c_{k}(0)$ as $k \rightarrow \infty$ effectively has been considered in [ 5 , p. 162]; see $\S 6$. It will be shown that although the growth of the terms in the expansion (1.1) is dominated by the familiar "factorial divided by a power" dependence, the coefficients also contain a multiplier function $f_{k}(\eta)$. In the present study, the behavior of this multiplier function is examined in detail, and in particular, we obtain a full asymptotic expansion for $f_{k}(\eta)$, involving the complementary error function, that is valid in a larger region of the $\eta$ plane than for which the original series (1.1) is valid. Considered as a function of the complex variable $\eta$, it is found that $f_{k}(\eta)$ is slowly varying in $\operatorname{Re}(\eta)>0$, but that in $\operatorname{Re}(\eta)<0$, there are two symmetrical lobes, with vertices at the origin and centered on the branch points $\eta=2 \sqrt{ } \pi \exp \left( \pm \frac{3}{4} \pi i\right)$, in which $f_{k}(\eta)$ becomes large. In a recent study of the coefficients appearing in the asymptotics of integrals which are dominated by coalescing distant saddle points, Berry and Howls [2] also observed a "decorated factorial series" behavior.

We present two different methods of determining the growth of the high-order coefficients $c_{k}(\eta)$. The first, given in $\S 2$, uses a treatment based on an integral representation for $c_{k}(\eta)$ followed by contour deformation and use of the saddle point method. This approach, which is described in $\S \S 3,4$ enables us to construct an asymptotic expansion for $c_{k}(\eta)$ as $k \rightarrow \infty$. In $\S 5$ (and the appendices), we show how to obtain an estimate for the behavior of $c_{k}(\eta)$ as $k \rightarrow \infty$ from their Maclaurin series expansion. Finally, in 
$\S 6$, we give numerical examples to compare the predictions of the asymptotic theory with numerically computed values of the high-order coefficients.

\section{An integral representation for $c_{k}(\eta)$}

We shall employ the representation (1.5) for $c_{k}(\eta)$. To overcome the difficulty of there being no simple representation for $Q_{k}(\mu)$, we proceed as follows. First, we write (for convenience)

$$
c_{k}(\eta)=\frac{(-2)^{k} \Gamma(\kappa)}{\Gamma\left(\frac{1}{2}\right)}\left\{\frac{\tilde{Q}_{k}(\mu)}{\mu^{2 \kappa}}-\frac{1}{\eta^{2 \kappa}}\right\}
$$

where

$$
\tilde{Q}_{k}(\mu)=\frac{\Gamma\left(\frac{1}{2}\right)}{2^{k} \Gamma(\kappa)} Q_{k}(\mu) .
$$

The right-hand side of (2.1) does not have a singularity at $\mu=0(\eta=0)$ since $c_{k}(\eta)$ is analytic at this point. Therefore, by Cauchy's integral formula, we can express the coefficients $c_{k}(\eta)$ in the form

$$
c_{k}(\eta)=\frac{(-2)^{k} \Gamma(\kappa)}{2 \pi i \Gamma\left(\frac{1}{2}\right)} \oint_{\mathcal{C}} \frac{1}{w-\mu}\left\{\frac{\tilde{Q}_{k}(w)}{w^{2 \kappa}}-\frac{1}{\hat{\eta}^{2 \kappa}}\right\} d w
$$

where

$$
\hat{\eta}=\{2(w-\ln (1+w))\}^{\frac{1}{2}}
$$

and the path of integration $\mathcal{C}$ is (initially) a simple closed curve lying in the slit plane $|\arg (w+1)|<\pi$, which encloses $w=\mu$; we temporarily assume that $|\arg (\mu+1)|<\pi$. For our purposes, we further require that $\mathcal{C}$ enclose $w=0$ (which is not a singularity of the integrand). We then expand the integrand to split the integral in (2.3) into two integrals: for the first of these (involving the polynomial $\tilde{Q}_{k}(\mu)$ ), the integrand has two singularities, at $w=0$ and $w=\mu$, and since both lie inside $\mathcal{C}$, we are permitted to expand the integration path to infinity, noting that the integrand is $O\left(w^{-2}\right)$ as $w \rightarrow \infty$. We conclude that the first integral is identically zero, and we are therefore left with the representation

$$
c_{k}(\eta)=-\frac{(-2)^{k} \Gamma(\kappa)}{2 \pi i \Gamma\left(\frac{1}{2}\right)} \oint_{\mathcal{C}} \frac{d w}{(w-\mu) \hat{\eta}^{2 \kappa}} .
$$

Note that the integrand in this formula now has an additional singularity at $\hat{\eta}=0$ $(w=0)$.

The next step is to make an appropriate deformation of $\mathcal{C}$. To do so, we make the simple change of variable $w \rightarrow t-1$ to yield

$$
c_{k}(\eta)=i \frac{(-)^{k} \Gamma(\kappa)}{(2 \pi)^{\frac{3}{2}}} I_{k}(\lambda)
$$

where

$$
I_{k}(\lambda)=\oint_{\mathcal{C}^{\prime}} \frac{d t}{(t-\lambda)(t-1-\ln (t))^{\kappa}} .
$$

In (2.7), $\mathcal{C}^{\prime}$ is a loop in the $t$ plane enclosing the poles $t=1$ and $t=\lambda$ (where we continue to assume that $|\arg (\lambda)|<\pi)$. Recall that the term $(t-1-\ln (t))^{-\kappa}$ is real and positive for $\arg (t)=0$ and $t>1$ and is defined by continuity elsewhere for 

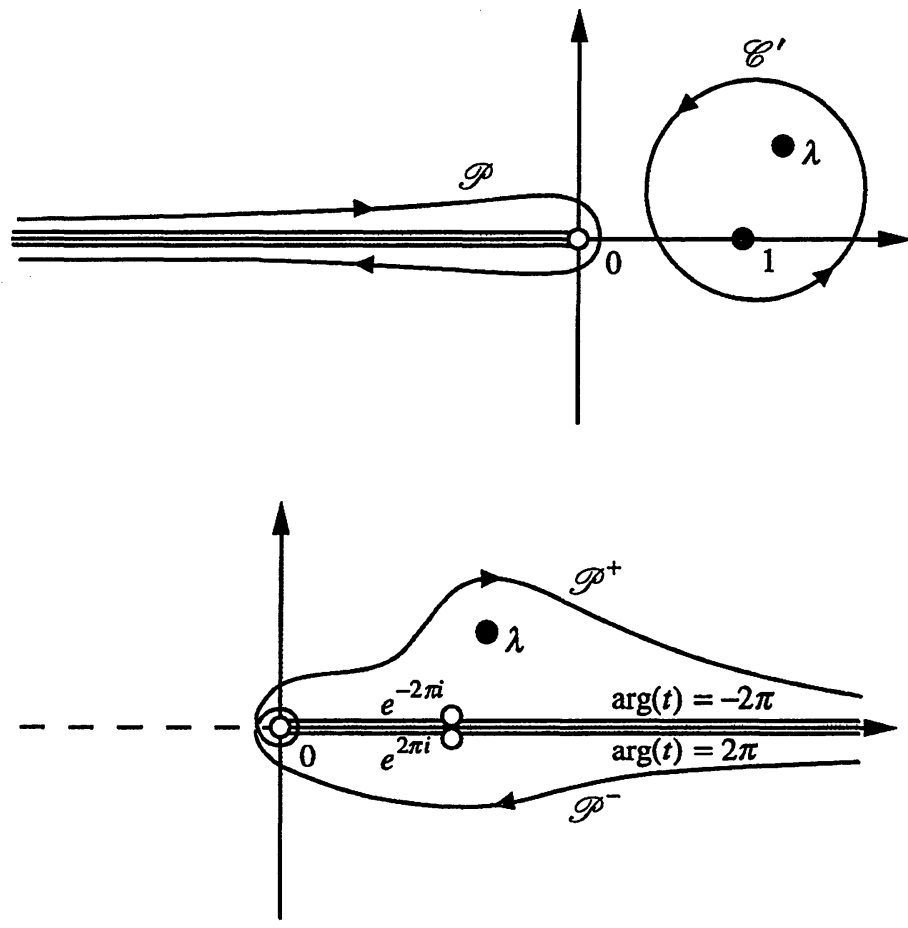

FIgURE 1. The contour of integration in the $t$ plane: (a) $-\pi \leq$ $\arg (t) \leq \pi$ and (b) $-2 \pi \leq \arg (t) \leq-\pi$ and $\pi \leq \arg (t) \leq 2 \pi$.

$-2 \pi \leq \arg (t) \leq 2 \pi$ (except, of course, at $t=1$ ). Note that the integrand is $O\left(t^{-k-\frac{3}{2}}\right)$ as $t \rightarrow \infty$ and, apart from the branch point at $t=0$ and poles at $t=1$ and $t=\lambda$, has no other singularities ${ }^{1}$ in the sheet $-2 \pi \leq \arg (t) \leq 2 \pi$.

We now first deform $\mathcal{C}^{\prime}$ into a loop $\mathcal{P}$ surrounding the branch point $t=0$ along the rays $\arg (t)= \pm \pi$ (see Figure 1a). Then we allow the component along $\arg (t)=\pi$ to pass into the half-plane $\pi \leq \arg (t) \leq 2 \pi$ and, likewise, the component along $\arg (t)=$ $-\pi$ to pass into the half-plane $-2 \pi \leq \arg (t) \leq-\pi$. As a result, we have deformed the path into one which envelops the branch point at $t=0$, with one component of $\mathcal{P}$ (which we call $\mathcal{P}^{-}$) extending from $t=\infty$ to $t=0$ with $\pi \leq \arg (t) \leq 2 \pi$ and the other, $\mathcal{P}^{+}$, from $t=0$ to $t=\infty$ with $-2 \pi \leq \arg (t) \leq-\pi$ (see Figure $1 \mathrm{~b}$ ). Since the branch point at $t=0$ is integrable, the loop around the origin yields no contribution. The integral (2.7) then can be expressed in the form

$$
I_{k}(\lambda)=I_{k}^{+}(\lambda)+I_{k}^{-}(\lambda)
$$

\footnotetext{
${ }^{1}$ The integrand in (2.7), however, does have singularities outside this sheet, for example, at $t=$ $\rho e^{2 \pi i}$ and $t=\bar{\rho} e^{-2 \pi i}$ where $\rho$ is the complex number in the first quadrant satisfying $\rho-1-\ln \rho=2 \pi i$ (to one significant figure, $\rho=3.1+7.5 i$ ). Since we shall not deform the contour outside the range $-2 \pi \leq \arg (t) \leq 2 \pi$, we shall not be concerned about these other singularities.
} 
where $I_{k}^{+}(\lambda)$ is given by the right-hand side of (2.7) but with the path of integration being the path from $t=0$ to $t=\infty$ with $-2 \pi \leq \arg (t) \leq-\pi$, and $I_{k}^{-}(\lambda)$ is similarly defined with the integration path running from $t=\infty$ to $t=0$ with $\pi \leq \arg (t) \leq 2 \pi$.

Consider the integral $I_{k}^{-}(\lambda)$. If we make the change of variable $t=\tau e^{2 \pi i}$, we obtain

$$
I_{k}^{-}(\lambda)=-\frac{1}{(-2 \pi i)^{\kappa}} \int_{\hat{\mathcal{P}}^{-}}\left\{1+\frac{i}{2 \pi}(\tau-1-\ln (\tau))\right\}^{-\kappa} \frac{d \tau}{\tau-\lambda}
$$

where $\hat{\mathcal{P}}^{-}$, the $\tau$ map of $\mathcal{P}^{-}$, extends from $\tau=\infty$ to $\tau=0$ in the right half of the principal $\tau$ plane, with the pole at $\tau=\lambda$ lying above $\hat{\mathcal{P}}^{-}$. Our task is now to investigate the asymptotic behavior of $I_{k}^{-}(\lambda)$ as $k \rightarrow \infty$; the treatment of $I_{k}^{+}(\lambda)$ is similar. Accordingly, we recast (2.9) into the standard form for obtaining a steepest descents approximation by making the further change of variable

$$
u=\{\ln [1+i(\tau-1-\ln (\tau)) /(2 \pi)]\}^{\frac{1}{2}},
$$

where $u$ is analytic at $\tau=1$, and the branch is chosen so that $u \sim e^{\frac{1}{4} \pi i}(\tau-1) /(2 \sqrt{ } \pi)$ as $\tau \rightarrow 1$. As a result, we obtain

$$
I_{k}^{-}(\lambda)=\frac{e^{\frac{1}{2} \pi i \kappa}}{(2 \pi)^{\kappa}} \int_{\mathcal{L}^{-}} \exp \left[-\kappa u^{2}\right] g^{-}(u, \lambda) \frac{d u}{u-u_{\lambda}^{-}}
$$

where we define the quantities $u_{\lambda}^{ \pm}$by

$$
u_{\lambda}^{ \pm}=\left\{\ln \left[1 \mp \frac{i}{2 \pi}(\lambda-1-\ln (\lambda))\right]\right\}^{\frac{1}{2}}=\left\{\ln \left(1 \mp \frac{i \eta^{2}}{4 \pi}\right)\right\}^{\frac{1}{2}}
$$

and

$$
g^{-}(u, \lambda)=\left(\frac{u-u_{\lambda}^{-}}{\tau-\lambda}\right) \frac{d \tau}{d u}
$$

The $u$ path $\mathcal{L}^{-}$corresponds to the $\tau$ path $\hat{\mathcal{P}}^{-}$with the direction reversed, so that $-\infty<\operatorname{Re} u<\infty$ as $u$ runs along $\mathcal{L}^{-}$.

Since $g^{-}(u, \lambda)$ is an analytic function of $u$ at $u_{\lambda}^{-}$, the integral (2.10) is characterized by having a pole at $u=u_{\lambda}^{-}$and, for large $k$, a saddle point at $u=0$. It is readily verified that $g^{-}(u, \lambda) \rightarrow 1$ as $u \rightarrow u_{\lambda}^{-}$, and as such we write

$$
g^{-}(u, \lambda)=1+\left(u-u_{\lambda}^{-}\right) G^{-}(u, \lambda)
$$

where $G^{-}(u, \lambda)$ is given by

$$
G^{-}(u, \lambda)=-\left\{\frac{1}{u-u_{\lambda}^{-}}+\left(\frac{4 \pi i \tau}{\tau-\lambda}\right)\left(\frac{u}{\tau-1}\right) \exp \left(u^{2}\right)\right\}
$$

and is analytic and non-vanishing at $u=u_{\lambda}^{-}$. On substituting (2.13) into (2.10), we arrive at

$$
I_{k}^{-}(\lambda)=\frac{e^{\frac{1}{2} \pi i \kappa}}{(2 \pi)^{\kappa}} \int_{\mathcal{L}^{-}} \exp \left[-\kappa u^{2}\right]\left\{\frac{1}{u-u_{\lambda}^{-}}+G^{-}(u, \lambda)\right\} d u .
$$

For the integral involving the first term in curly brackets, we use the formula

$$
\int_{\mathcal{L}^{-}} \exp \left(-\omega t^{2}\right) \frac{d t}{t-a}= \pm \pi i e^{-\omega a^{2}} \operatorname{erfc}(\mp i a \sqrt{ } \omega)
$$


where the upper signs are taken when the complex number $a$ lies above the contour $\mathcal{L}^{-}$, and the lower signs are taken otherwise. It then follows that

$$
I_{k}^{-}(\lambda)=\frac{\pi i e^{\frac{1}{2} \pi i \kappa}}{(2 \pi)^{\kappa}}\left\{\exp \left[-\kappa\left(u_{\lambda}^{-}\right)^{2}\right] \operatorname{erfc}\left(-i \sqrt{ } \kappa u_{\lambda}^{-}\right)+J_{k}^{-}(\lambda)\right\}
$$

where

$$
J_{k}^{-}(\lambda)=-\frac{i}{\pi} \int_{\mathcal{L}^{-}} \exp \left[-\kappa u^{2}\right] G^{-}(u, \lambda) d u .
$$

By a similar treatment, we find that

$$
I_{k}^{+}(\lambda)=\overline{I_{k}^{-}(\bar{\lambda})}
$$

where the bar denotes the complex conjugate. Consequently, we deduce from (2.6), (2.8), (2.16), and (2.18) that

$$
c_{k}(\eta)=\frac{\Gamma(\kappa)}{(-2 \pi)^{k+1}}\left\{f_{k}^{+}(\eta)+f_{k}^{-}(\eta)\right\},
$$

where, at this point, we have found it convenient to separate out the fast $k$-dependence of $c_{k}(\eta)$ by introducing the scaled multipliers $f_{k}^{ \pm}(\eta)$. Recalling from (1.2) and (1.3) that $\lambda=\lambda(\eta)$, we find

$$
f_{k}^{ \pm}(\eta)=\frac{1}{2} e^{\mp \frac{1}{2} \pi i \kappa}\left\{\left(1 \mp \frac{i \eta^{2}}{4 \pi}\right)^{-\kappa} \operatorname{erfc}\left( \pm i \sqrt{ } \kappa u_{\lambda}^{ \pm}\right)+J_{k}^{ \pm}(\lambda)\right\}
$$

in which $u_{\lambda}^{ \pm}$and $J_{k}^{-}(\lambda)$ are given by (2.11) and (2.17), and

$$
J_{k}^{+}(\lambda)=\overline{J_{k}^{-}(\bar{\lambda})}
$$

From this last result it therefore follows that $f_{k}^{+}(\eta)=\overline{f_{k}^{-}(\bar{\eta})}$.

Since the integrand of (2.17) is free of singularity at $u=u_{\lambda}^{-}$, we can deform the integration path $\mathcal{L}^{-}$to the one corresponding to $\arg (\tau)=0$ with $0 \leq \tau<\infty$. By analytic continuation, our restriction $|\arg (\lambda)|<\pi$ now can be relaxed to $|\arg (\lambda)|<$ $2 \pi-\delta$ where $\delta$ is an arbitrary small positive constant. This is because $G^{-}(u, \lambda)$, as defined by (2.14), is an analytic function of $\lambda$ in $|\arg (\lambda)|<2 \pi-\delta$ for each fixed $\tau$ (and corresponding $u$ ) with $\arg (\tau)=0$. However, if $|\arg (\lambda)|=2 \pi, G^{-}(u, \lambda)$ is singular at the point $u=u_{|\lambda|}^{-}$on the integration path (which corresponds to $\tau=|\lambda|$ ). To see this, we observe that $u_{|\lambda|}^{-} \neq u_{\lambda}^{-}$(see (2.11)), and hence the first term on the right-hand side of (2.14) is no longer a pole ${ }^{2}$ at $u=u_{|\lambda|}^{-}$, whereas the second term remains a pole at $\tau=|\lambda|$ since the factor $(\tau-\lambda)^{-1}$ is clearly single-valued. Extension beyond the range $|\arg (\lambda)|<2 \pi-\delta$ may be possible for certain values of $|\lambda|$ via a suitable further deformation of integration path, but we do not investigate such an extension since the stated region of validity is larger than that of the original asymptotic expansion (1.1).

So far our analysis has been exact. We now focus on the asymptotics of $J_{k}^{-}(\lambda)$ as $k \rightarrow \infty$. From the definition of $u$, it is straightforward to show that $\operatorname{Re} u^{2}>0$ on the new path described above, except at the saddle $u=0$ (which lies on the path), and hence the method of steepest descents can be applied. By taking the leading term

\footnotetext{
${ }^{2}$ Recall that when $\arg (\lambda)=0$, the poles in (2.14) cancel at $u=u_{\lambda}^{-}$to yield analyticity.
} 
in the standard saddle point asymptotics of the integrals $J_{k}^{-}(\lambda)$ (see, for example, $[8$, Chap. $4, \S 7])$, and referring to $(2.21)$, we arrive at

$$
f_{k}^{ \pm}(\eta)=\frac{1}{2} e^{\mp \frac{1}{2} \pi i \kappa}\left\{\left(1 \mp \frac{i \eta^{2}}{4 \pi}\right)^{-\kappa} \operatorname{erfc}\left( \pm i \sqrt{ } \kappa u_{\lambda}^{ \pm}\right) \pm i \frac{\Gamma\left(\frac{1}{2}\right)}{\pi \sqrt{ } \kappa} a_{0}^{ \pm}(\mu)\right\}
$$

as $k \rightarrow \infty$ where $($ for $\mu \neq 0$ )

$$
a_{0}^{ \pm}(\mu)=\frac{1}{u_{\lambda}^{ \pm}}-\frac{2 \sqrt{ } \pi e^{ \pm \frac{1}{4} \pi i}}{\mu} .
$$

The expressions in (2.22) hold in the limit as $\mu \rightarrow 0(\eta \rightarrow 0)$, and one finds in this case that

$$
a_{0}^{ \pm}(0)=\frac{2 \sqrt{ } \pi}{3} e^{ \pm \frac{1}{4} \pi i}
$$

\section{Asymptotic expansion}

We now obtain an asymptotic expansion for the coefficients $c_{k}(\eta)$ as $k \rightarrow \infty$, which entails deriving higher terms in the saddle point expansions for $J_{k}^{ \pm}(\lambda)$. From the relation (2.21), it suffices to consider only one of these integrals, namely $J_{k}^{+}(\lambda)$, which is given by

$$
J_{k}^{+}(\lambda)=\frac{i}{\pi} \int_{\mathcal{L}^{+}} \exp \left[-\kappa u^{2}\right] G^{+}(u, \lambda) d u
$$

where now $u=\{\ln [1-i(\tau-1-\ln (\tau)) /(2 \pi)]\}^{\frac{1}{2}}$,

$$
G^{+}(u, \lambda)=\left(\frac{4 \pi i \tau}{\tau-\lambda}\right)\left(\frac{u}{\tau-1}\right) \exp \left(u^{2}\right)-\frac{1}{u-u_{\lambda}^{+}},
$$

$u_{\lambda}^{+}$is given by (2.11), and the path of integration $\mathcal{L}^{+}$corresponds to $\tau$ lying on the positive real axis (with $\arg (\tau)=0$ ). From an application of the general saddle point method, with higher order terms, we immediately find an expansion of the form

$$
J_{k}^{+}(\lambda) \sim \frac{i}{\pi} \sum_{m=0}^{\infty} \frac{\Gamma\left(m+\frac{1}{2}\right) a_{m}^{+}(\mu)}{\kappa^{m+\frac{1}{2}}}
$$

where the coefficients $a_{m}^{+}(\mu)$ are formally generated from the coefficients appearing in the Maclaurin expansion (as a function of $u$ ) of $G^{+}(u, \lambda)$. Unfortunately, the technique for generating these coefficients directly from the saddle point theory is rather complicated and yields no insight into their general form. The purpose of this section is to address this shortcoming.

First, it is straightforward to verify by induction that each coefficient $a_{m}^{+}(\mu), m=$ $0,1,2, \ldots$ has the following general structure

$$
a_{m}^{+}(\mu)=\left(u_{\lambda}^{+}\right)^{-2 m-1}-\left\{\frac{A_{2 m+1}^{(m)}}{\mu^{2 m+1}}+\frac{A_{2 m}^{(m)}}{\mu^{2 m}}+\cdots+\frac{A_{1}^{(m)}}{\mu}\right\}
$$

where $A_{j}^{(m)}$ are (complex) coefficients which are independent of $\mu$. We do not attempt to calculate these numbers directly, but rather re-expand the $(2 m+1)$-degree polynomial in $\mu^{-1}$ on the right-hand side of (3.4) (in curly brackets) in terms of the polynomials $\tilde{Q}_{k}(\mu)$ (which are defined by $(2.2)$ ). The technique is to observe that since $a_{m}^{+}(\mu)$ is analytic at $\mu=0$, the singularities at this point of $\left(u_{\lambda}^{+}\right)^{-2 m-1}$ and the polynomial in $\mu^{-1}$ on the right-hand side of (3.4) must cancel. 
With the above observations in mind, we expand $\left(u_{\lambda}^{+}\right)^{-2 m-1}$ as follows. First, by differentiation with respect to $x$, it is verifiable that near $x=0$

$$
\left\{\frac{x}{\ln (1+x)}\right\}^{m+\frac{1}{2}}=\sum_{j=0}^{\infty} b_{j}^{(m)} x^{j}
$$

where ${ }^{3}$

$$
\begin{gathered}
b_{0}^{(m)}=1, \quad b_{1}^{(m)}=\frac{1}{2} \sigma \\
b_{j}^{(m)}=(-)^{j+1} \frac{\sigma}{j+1}+\frac{1}{j} \sum_{k=1}^{j-1}(-)^{k+1} \frac{\{(\sigma-1) k+j\}}{k+1} b_{j-k}^{(m)}, \quad(j \geq 2),
\end{gathered}
$$

with $\sigma=m+\frac{1}{2}$. Therefore, for small $|\eta|$ (or, equivalently, small $|\mu|$ ), it follows that

$$
\left(u_{\lambda}^{+}\right)^{-2 m-1}=\left\{\ln \left(1-\frac{i \eta^{2}}{4 \pi}\right)\right\}^{-m-\frac{1}{2}}=(h \eta)^{-2 m-1}\left\{b_{0}^{(m)}+b_{1}^{(m)}(h \eta)^{2}+b_{2}^{(m)}(h \eta)^{4}+\cdots\right\}
$$

where $h=e^{-\frac{1}{4} \pi i} /(2 \sqrt{ } \pi)$. Next, since $c_{k}(\eta)$ is analytic at $\eta=0(\mu=0)$ for $k=$ $0,1,2, \ldots$, we deduce from $(2.1)$ that

$$
\frac{1}{\eta^{2 \kappa}}=\frac{\tilde{Q}_{k}(\mu)}{\mu^{2 \kappa}}+O(1)
$$

as $\mu \rightarrow 0(\eta \rightarrow 0)$. On substituting (3.7) into the series for $\left(u_{\lambda}^{+}\right)^{-2 m-1}$ given by (3.6), we then find that

$$
\left(u_{\lambda}^{+}\right)^{-2 m-1}=\sum_{j=0}^{m} b_{m-j}^{(m)} \frac{\tilde{Q}_{j}(\mu)}{(h \mu)^{2 j+1}}+O(1) .
$$

Analogously, from (3.4), we can assert that as $\mu \rightarrow 0$

$$
\left(u_{\lambda}^{+}\right)^{-2 m-1}=\left\{\frac{A_{2 m+1}^{(m)}}{\mu^{2 m+1}}+\frac{A_{2 m}^{(m)}}{\mu^{2 m}}+\cdots+\frac{A_{1}^{(m)}}{\mu}\right\}+O(1)
$$

since $a_{m}^{+}(\mu)$ is also analytic at $\mu=0$. On comparing (3.8) with (3.9), it then must follow that

$$
\left\{\frac{A_{2 m+1}^{(m)}}{\mu^{2 m+1}}+\frac{A_{2 m}^{(m)}}{\mu^{2 m}}+\cdots+\frac{A_{1}^{(m)}}{\mu}\right\}-\sum_{j=0}^{m} b_{m-j}^{(m)} \frac{\tilde{Q}_{j}(\mu)}{(h \mu)^{2 j+1}}=O(1) .
$$

However, the $\mathrm{O}(1)$ term on the right-hand side of this equation must actually be identically zero, since the left-hand side is a polynomial in $\mu^{-1}$ which does not contain a constant term; recall that $\tilde{Q}_{j}(\mu)$ is a polynomial in $\mu$ of order $2 j$. We therefore have proved that

$$
\frac{A_{2 m+1}^{(m)}}{\mu^{2 m+1}}+\frac{A_{2 m}^{(m)}}{\mu^{2 m}}+\cdots+\frac{A_{1}^{(m)}}{\mu}=\sum_{j=0}^{m} b_{m-j}^{(m)} \frac{\tilde{Q}_{j}(\mu)}{(h \mu)^{2 j+1}}
$$

\footnotetext{
${ }^{3}$ The coefficients $b_{j}^{(m)}$ can be expressed alternatively in the form $b_{j}^{(m)}=(1 / j !) \sum_{r=0}^{j} B_{r}^{(\sigma)}(0) S_{j}^{(r)}$ where $B_{r}^{(\sigma)}(x)$ is the generalized Bernoulli polynomial and $S_{j}^{(r)}$ is the Stirling number of the first kind.
} 
The final step is to substitute (3.10) into (3.4), and then in this expression for $a_{m}^{+}(\mu)$ to use (2.1) to write

$$
\frac{\tilde{Q}_{j}(\mu)}{\mu^{2 j+1}}=\frac{1}{\eta^{2 j+1}}+\frac{\sqrt{ } \pi}{(-2)^{j} \Gamma\left(j+\frac{1}{2}\right)} c_{j}(\eta) .
$$

As a result, we arrive at our desired representation

$$
a_{m}^{+}(\mu)=\left(u_{\lambda}^{+}\right)^{-2 m-1}-2 \pi e^{\frac{1}{4} \pi i} \sum_{j=0}^{m}(4 \pi i)^{j} b_{m-j}^{(m)}\left\{\frac{1}{\sqrt{ } \pi \eta^{2 j+1}}+\frac{c_{j}(\eta)}{(-2)^{j} \Gamma\left(j+\frac{1}{2}\right)}\right\} .
$$

The analysis of $J_{k}^{-}(\lambda)$ is similar (cf. (2.21)) and leads to the coefficients $a_{m}^{ \pm}(\mu)$, which we rewrite in the form

$$
a_{m}^{ \pm}(\mu)=\mathcal{A}_{m}^{ \pm}(\eta)+e^{\mp \frac{1}{4} \pi i} \sum_{j=0}^{m}(-2 \pi i)^{j+1} \frac{b_{m-j}^{(m)}}{\Gamma\left(j+\frac{1}{2}\right)} c_{j}(\eta)
$$

where

$$
\mathcal{A}_{m}^{ \pm}(\eta)=\left(u_{\lambda}^{ \pm}\right)^{-2 m-1}-\left(\mp \frac{i \eta^{2}}{4 \pi}\right)^{-m-\frac{1}{2}} \sum_{j=0}^{m} b_{j}^{(m)}\left(\mp \frac{i \eta^{2}}{4 \pi}\right)^{j}
$$

We note that the quantities $\mathcal{A}_{m}^{ \pm}(\eta)$ correspond to the removal of the first $m+1$ terms from the series expansion (valid in $|\eta|<2 \sqrt{ } \pi$ ) of $\left(u_{\lambda}^{ \pm}\right)^{-2 m-1}$ in (3.6), and consequently that $\mathcal{A}_{m}^{ \pm}(0)=0$.

We now have our main result:

Theorem 3.1. With the notation of $\S \S 1,2$ and the present section

$$
c_{k}(\eta)=\frac{\Gamma(\kappa)}{(-2 \pi)^{k+1}}\left\{f_{k}^{+}(\eta)+f_{k}^{-}(\eta)\right\}
$$

where $f_{k}^{ \pm}(\eta)$ have the asymptotic expansions as $k \rightarrow \infty$

$$
f_{k}^{ \pm}(\eta) \sim \frac{1}{2} e^{\mp \frac{1}{2} \pi i \kappa}\left\{\left(1 \mp \frac{i \eta^{2}}{4 \pi}\right)^{-\kappa} \operatorname{erfc}\left( \pm i \sqrt{ } \kappa u_{\lambda}^{ \pm}\right) \pm \frac{i}{\pi} \sum_{m=0}^{\infty} \frac{\Gamma\left(m+\frac{1}{2}\right) a_{m}^{ \pm}(\mu)}{\kappa^{m+\frac{1}{2}}}\right\}
$$

with $\kappa=k+\frac{1}{2}$ and coefficients given by

$$
a_{m}^{ \pm}(\mu)=\mathcal{A}_{m}^{ \pm}(\eta)+e^{\mp \frac{1}{4} \pi i} \sum_{j=0}^{m}(\mp 2 \pi i)^{j+1} \frac{b_{m-j}^{(m)}}{\Gamma\left(j+\frac{1}{2}\right)} c_{j}(\eta) .
$$

The quantities $u_{\lambda}^{ \pm}$and $\mathcal{A}_{m}^{ \pm}(\eta)$ are given in (2.11) and (3.12), respectively, and the coefficients $b_{j}^{(m)}$ are defined by the recursion (3.5). The expansion (3.14) is valid for all $\eta$ values corresponding to $|\arg (\lambda)|<2 \pi-\delta$ where $\delta$ is an arbitrary small positive constant. 


\section{Discussion of the asymptotic behavior}

We examine the behavior of $c_{k}(\eta)$ from the relation (3.13) and the asymptotic result (3.14). We shall show the existence of two lobes, in the second and third quadrants of the $\eta$ plane, in which $c_{k}(\eta)$ is exponentially large, as $k \rightarrow \infty$, relative to its values elsewhere in the complex $\eta$ plane.

First, it is well known that (in the Poincaré sense) as $z \rightarrow \infty$,

$$
\operatorname{erfc}(z) \sim \begin{cases}e^{-z^{2}} /(\sqrt{ } \pi z) & \left(|\arg (z)| \leq \frac{3}{4} \pi-\delta\right) \\ 2+e^{-z^{2}} /(\sqrt{ } \pi z) & \left(|\arg (-z)| \leq \frac{3}{4} \pi-\delta\right)\end{cases}
$$

where $\delta>0$. Suppose first that $\left|\arg \left( \pm i u_{\lambda}^{ \pm}\right)\right| \leq \frac{3}{4} \pi-\delta$. Then, from (2.23) and (3.15) the coefficients $a_{0}^{ \pm}(\mu)$ and $a_{1}^{ \pm}(\mu)$ are given by

$$
\begin{aligned}
& a_{0}^{ \pm}(\mu)=\frac{1}{u_{\lambda}^{ \pm}}-\frac{2 \sqrt{ } \pi}{\mu} e^{ \pm \frac{1}{4} \pi i} \\
& a_{1}^{ \pm}(\mu)=\frac{1}{\left(u_{\lambda}^{ \pm}\right)^{3}}-\frac{3 \sqrt{ } \pi}{2 \mu} e^{ \pm \frac{1}{4} \pi i}\left(1 \pm \frac{16 \pi i}{3 \mu^{2}} Q_{1}(\mu)\right),
\end{aligned}
$$

so that, from (4.1), (3.14), and the definition (2.11), it follows that

$$
f_{k}^{ \pm}(\eta)=\frac{e^{\mp \frac{1}{2} \pi i \kappa \mp \frac{1}{4} \pi i}}{\mu \sqrt{ } \kappa}\left(1+\frac{3}{8 \kappa} \pm \frac{2 \pi i}{\mu^{2} \kappa} Q_{1}(\mu)+O\left(\kappa^{-2}\right)\right)
$$

for fixed $\mu \neq 0$. We then obtain from (3.13)

$$
c_{k}(\eta)=\frac{\Gamma(\kappa)}{(-2 \pi)^{k+1}}\left\{-\frac{2}{\mu \sqrt{ } \kappa}\left(1+\frac{3}{8 \kappa}\right) \sin \left(\frac{1}{2} \pi k\right)+\frac{4 \pi}{\mu^{3} \kappa^{\frac{3}{2}}} Q_{1}(\mu) \cos \left(\frac{1}{2} \pi k\right)+O\left(\kappa^{-\frac{5}{2}}\right)\right\}
$$

as $k \rightarrow \infty$.

On the other hand, if $\left|\arg \left(\mp i u_{\lambda}^{ \pm}\right)\right| \leq \frac{1}{4} \pi-\delta$ and $k \rightarrow \infty$, then, from (4.1),

$$
f_{k}^{ \pm}(\eta)=e^{\mp \frac{1}{2} \pi i \kappa}\left\{\left(1 \mp \frac{i \eta^{2}}{4 \pi}\right)^{-\kappa}+\frac{e^{\mp \frac{1}{4} \pi i}}{\mu \sqrt{ } \kappa}\left(1+\frac{3}{8 \kappa}\right)+\frac{2 \pi}{\mu^{3} \kappa^{\frac{3}{2}}} e^{ \pm \frac{1}{4} \pi i} Q_{1}(\mu)+O\left(\kappa^{-\frac{5}{2}}\right)\right\} \text {. }
$$

Now since $\left(1 \mp i \eta^{2} /(4 \pi)\right)^{-\kappa}=\exp \left\{-\kappa\left(u_{\lambda}^{ \pm}\right)^{2}\right\}$, it is seen that the terms $f_{k}^{ \pm}(\eta)$ are exponentially large as $k \rightarrow \infty$ in the sectors $\left|\arg \left(\mp i u_{\lambda}^{ \pm}\right)\right| \leq \frac{1}{4} \pi-\delta$, respectively. Thus, when $\left|\arg \left(-i u_{\lambda}^{+}\right)\right| \leq \frac{1}{4} \pi-\delta$, the term $f_{k}^{+}(\eta)$ is dominant and

$$
c_{k}(\eta) \sim \frac{e^{-\frac{1}{2} \pi i \kappa} \Gamma(\kappa)}{(-2 \pi)^{k+1}}\left(1-\frac{i \eta^{2}}{4 \pi}\right)^{-\kappa}
$$

and when $\left|\arg \left(i u_{\lambda}^{-}\right)\right| \leq \frac{1}{4} \pi-\delta$, the term $f_{k}^{-}(\eta)$ is dominant, leading to

$$
c_{k}(\eta) \sim \frac{e^{\frac{1}{2} \pi i \kappa} \Gamma(\kappa)}{(-2 \pi)^{k+1}}\left(1+\frac{i \eta^{2}}{4 \pi}\right)^{-\kappa}
$$

The regions $\left|\arg \left(\mp i u_{\lambda}^{ \pm}\right)\right| \leq \frac{1}{4} \pi$ correspond to $\eta$ lying in certain domains $\mathbf{L}^{ \pm}$, say, in the second and third quadrants, respectively. These two lobes (including their maps in the $\lambda$ plane), in which $c_{k}(\eta)$ is exponentially large as $k \rightarrow \infty$, are indicated in Figure 2. It can be shown that $\mathbf{L}^{ \pm}$correspond to one leaf of the lemniscates $|\eta|^{2} \pm 8 \pi \sin (2 \theta)=0$ where $\theta=\arg (\eta)$. 


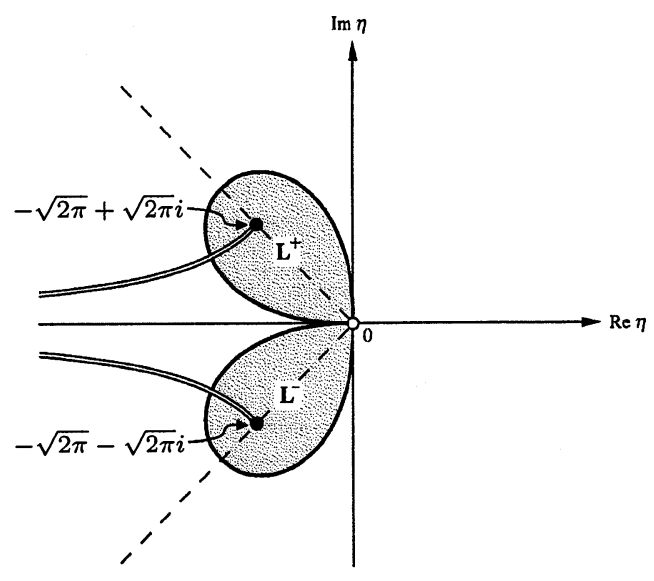

(a)

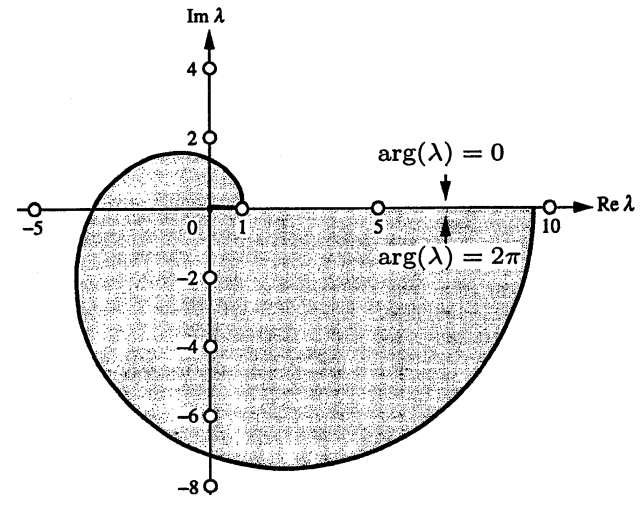

(b)

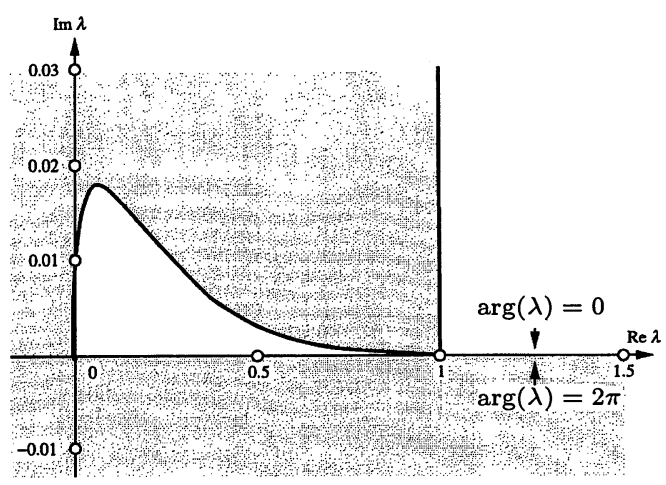

(c)

Figure 2. (a) The lobes of dominance $\mathbf{L}^{ \pm}$in the $\eta$ plane and (b), (c) the map of $\mathbf{L}^{+}$in the $\lambda$ plane. The boundaries in (b) and (c) correspond to the boundaries of $\mathbf{L}^{+}$which emanate from $\eta=0$ and reach the upper and lower sides of the cut, respectively. Even under the magnification in (c), it is not possible to discern that $\lambda=0$, together with some small neighborhood, is excluded from the map of $\mathbf{L}^{+}$. The map of $\mathbf{L}^{-}$is given by the conjugate domain. The branch cuts in the $\eta$ plane correspond to the cuts on $\arg \lambda= \pm 2 \pi$.

We note that the presence of singularities in (4.5) and (4.6) (in the second and third quadrants) at $\eta=2 \sqrt{ } \pi e^{ \pm \frac{3}{4} \pi i}$ (corresponding to $\lambda=e^{ \pm 2 \pi i}$ ) is explained by the non-analyticity of $c_{k}(\eta)$ at those points. 


\section{Maclaurin series expansion}

In this section, we show how the large $k$ behavior of the coefficients $c_{k}(\eta)$ can be obtained alternatively from their Maclaurin series expansion. The series expansion of the coefficients $c_{k}(\eta)$ defined in (1.4) is given by

$$
c_{k}(\eta)=\sum_{n=0}^{\infty} \beta_{n k} \eta^{n} \quad(|\eta|<2 \sqrt{ } \pi)
$$

where the coefficients $\beta_{n k}$ can be written in the form [13, Eq. (3.9)]

$$
\beta_{n k}=\sum_{r=0}^{k} \beta_{n k}^{(r)}, \quad \beta_{n k}^{(r)}=2^{\delta} \gamma_{r} \frac{\Gamma\left(\frac{1}{2} n+\delta+1\right)}{\Gamma\left(\frac{1}{2} n+1\right)} \alpha_{n+2 \delta}, \quad \delta=k-r+1 .
$$

The coefficients $\alpha_{k}$ are those in the expansion of $\mu(\eta)$ given by

$$
\mu=\eta+\sum_{n=2}^{\infty} \alpha_{n} \eta^{n} \quad(|\eta|<2 \sqrt{ } \pi),
$$

and $\gamma_{k}$ are the Stirling coefficients in (1.6). The finite radius of convergence of (5.1) and (5.3) results from the presence of branch points situated at $\eta=2 \sqrt{ } \pi \exp \left( \pm \frac{3}{4} \pi i\right)$; see below. Substitution of (5.2) in (5.1) then enables us to express $c_{k}(\eta)$ in terms of the partial sums

$$
c_{k}(\eta)=\sum_{r=0}^{k} c_{k}^{(r)}(\eta), \quad c_{k}^{(r)}(\eta)=\sum_{n=0}^{\infty} \beta_{n k}^{(r)} \eta^{n} \quad(|\eta|<2 \sqrt{ } \pi) .
$$

It is possible to relate the $r$ th partial sum, $c_{k}^{(r)}(\eta)$, to the coefficients $c_{j}(\eta)(j=$ $0,1, \ldots, k-r)$ and thereby invert the finite sum in (5.4). This is carried out in Appendix A, where it is shown in particular that the partial sum $c_{k}^{(0)}(\eta)$, obtained by choosing $r=0$ in (A.1), satisfies the relation

$$
c_{k}^{(0)}(\eta)=\gamma_{0} \sum_{j=0}^{k}(-)^{j} \gamma_{j} c_{k-j}(\eta) \quad(k \geq 1)
$$

where $\gamma_{0}=1$. We now scale out the fast $k$-dependence of $c_{k}(\eta)$, as in (2.19), by defining

$$
c_{k}(\eta)=\frac{\Gamma(\kappa)}{(-2 \pi)^{k+1}} f_{k}(\eta)
$$

and similarly for $c_{k}^{(0)}(\eta)$ in terms of the scaled multiplier $f_{k}^{(0)}(\eta)$. Then (5.5) takes the form

$$
f_{k}(\eta)=f_{k}^{(0)}(\eta)-\sum_{j=1}^{k}(2 \pi)^{j} \frac{\Gamma(\kappa-j)}{\Gamma(\kappa)} \gamma_{j} f_{k-j}(\eta)
$$

where $\kappa$ is defined in (1.5). The identity (5.7) will form the basis for the determination of the asymptotic behavior of $f_{k}(\eta)$ as $k \rightarrow \infty$.

We first consider the function $f_{k}^{(0)}(\eta)$, which by (5.4), (5.2), and (5.6), is given by

$$
f_{k}^{(0)}(\eta)=\frac{(-4 \pi)^{k+1}}{\Gamma(\kappa)} \sum_{n=0}^{\infty} \alpha_{n+2 k+2} \frac{\Gamma\left(\frac{1}{2} n+k+2\right)}{\Gamma\left(\frac{1}{2} n+1\right)} \eta^{n}, \quad(|\eta|<2 \sqrt{ } \pi) .
$$


The large $k$ behavior of $f_{k}^{(0)}(\eta)$ is derived in Appendix B, where it is shown that (see (B.2)-(B.4))

$$
f_{k}^{(0)}(0)=(-)^{k}\left\{\sin \left(\frac{1}{2} \pi \kappa\right)-\frac{\pi}{6 k} \cos \left(\frac{1}{2} \pi \kappa\right)+O\left(k^{-2}\right)\right\}, \quad k \rightarrow \infty,
$$

and when $|\eta|$ is finite and bounded away from zero (but not limited to the disc $|\eta|<$ $2 \sqrt{ } \pi)($ see $($ B.8) $)$

$$
f_{k}^{(0)}(\eta) \sim \begin{cases}D_{k}(\eta) & \left(|\arg (\eta)| \leq \frac{1}{2} \pi\right) \\ D_{k}(\eta)+E_{k}^{\mp}(\eta) & \left(\frac{1}{2} \pi<|\arg (\eta)| \leq \pi\right)\end{cases}
$$

where

$$
\begin{gathered}
D_{k}(\eta)=\frac{2(-)^{k}}{\eta \sqrt{ } k}\left\{\left(1+\frac{3}{8 k}\right) \sin \left(\frac{1}{2} \pi k\right)-\frac{\pi}{6 k}\left(1-\frac{12}{\eta^{2}}\right) \cos \left(\frac{1}{2} \pi k\right)+O\left(k^{-2}\right)\right\} \\
E_{k}^{ \pm}(\eta)=e^{ \pm \frac{1}{2} \pi i \kappa}\left(1 \pm \frac{i \eta^{2}}{4 \pi}\right)^{-\kappa}\left\{1+O\left(k^{-1}\right)\right\}
\end{gathered}
$$

and the upper and lower signs are chosen according as $\eta$ lies in the second or third quadrants, respectively.

If we now assume that $f_{k}(\eta)$ possesses a similar dependence on $k$ to that in $(5.10)$ (this can be verified a posteriori or by appeal to the results in $\S 2$ ), then because of the inverse factorial nature of the sum in (5.7) and the well-known behavior of the Stirling coefficients for $k \rightarrow \infty[5$, p. $159 ; 3]$,

$$
\gamma_{k}=\left\{\begin{array}{ll}
\frac{1}{\pi}(-)^{(k+1) / 2} \frac{\Gamma(k)}{(2 \pi)^{k}}\left\{1+O\left(k^{-1}\right)\right\} & (k \text { odd }) \\
-\frac{1}{6}(-)^{k / 2} \frac{\Gamma(k-1)}{(2 \pi)^{k}}\left\{1+O\left(k^{-1}\right)\right\} & (k \text { even })
\end{array},\right.
$$

the terms in this sum for large $k$ at first decrease rapidly in absolute value and then subsequently increase. As a consequence, it is found that for the algebraic component, the terms corresponding to $j=k$ (when $k$ is odd) and to $j=1, k-1$, and $k$ (when $k$ is even) contribute ${ }^{4}$ to the leading behavior of $f_{k}(\eta)$, while for the exponentially large component, the sum (5.7) does not contribute to leading order. Thus, we find as $k \rightarrow \infty$

$$
f_{k}(\eta)=f_{k}^{(0)}(\eta)+ \begin{cases}\frac{(2 \pi)^{k+1}}{\Gamma(\kappa)} \gamma_{k} c_{0}(\eta) & (k \text { odd }) \\ \frac{(2 \pi)^{k+1}}{\Gamma(\kappa)}\left\{\gamma_{k} c_{0}(\eta)-\gamma_{k-1} c_{1}(\eta)\right\}-\frac{2 \pi \gamma_{1}}{k-\frac{1}{2}} f_{k-1}(\eta) & (k \text { even })\end{cases}
$$

Use of the coefficients $c_{0}(\eta)=1 / \mu-1 / \eta, c_{1}(\eta)=1 / \eta^{3}-Q_{1}(\mu) / \mu^{3}$ (see (1.5)) where $Q_{1}(\mu)=1+\mu+\mu^{2} / 12$, and (5.11) then produces the leading behavior of $f_{k}(\eta)$ for $k \rightarrow \infty$ in the form

$$
f_{k}(\eta) \sim \begin{cases}(-)^{(k+1) / 2} 2 /\left(\mu k^{\frac{1}{2}}\right) & \left(|\arg (\eta)| \leq \frac{1}{2} \pi\right) \\ (-)^{(k+1) / 2} 2 /\left(\mu k^{\frac{1}{2}}\right)+E_{k}^{\mp}(\eta) & \left(\frac{1}{2} \pi<|\arg (\eta)| \leq \pi\right)\end{cases}
$$

\footnotetext{
${ }^{4}$ The dominant terms in the sum (5.7) for even and odd $k$ are $O\left(k^{-\frac{3}{2}}\right)$ and $O\left(k^{-\frac{1}{2}}\right)$, respectively.
} 
when $k$ is odd, and

$$
f_{k}(\eta) \sim \begin{cases}(-)^{k / 2} 4 \pi Q_{1}(\mu) /\left(\mu^{3} k^{\frac{3}{2}}\right) & \left(|\arg (\eta)| \leq \frac{1}{2} \pi\right) \\ (-)^{k / 2} 4 \pi Q_{1}(\mu) /\left(\mu^{3} k^{\frac{3}{2}}\right)+E_{k}^{\mp}(\eta) & \left(\frac{1}{2} \pi<|\arg (\eta)| \leq \pi\right)\end{cases}
$$

when $k$ is even, valid when $|\eta|$ is bounded away from zero. The behavior of $f_{k}(\eta)$ when $\eta=0$ can be found by substituting (5.9) into (5.12) to find, as $k \rightarrow \infty$,

$$
f_{k}(0) \sim(-)^{k} \sin \left(\frac{1}{2} \pi \kappa\right)+ \begin{cases}(-)^{(k-1) / 2} 2 /\left(3 k^{\frac{1}{2}}\right) & (k \text { odd }) \\ (-)^{k / 2} \pi /\left(135 k^{\frac{3}{2}}\right) & (k \text { even })\end{cases}
$$

where we have employed the values $c_{0}(0)=-\frac{1}{3}$ and $c_{1}(0)=-\frac{1}{540}$.

The leading forms (5.13)-(5.15) can be seen to agree with the results derived in $\S 4$ from the more refined analysis of the scaled multiplier function based on an integral representation for $c_{k}(\eta)$. Thus, when $k \rightarrow \infty$, the approximations given by the first equations in (5.13) and (5.14) continue to describe the dominant behavior of $f_{k}(\eta)$ in $\operatorname{Re}(\eta)<0$, except when $\eta\left(=|\eta| e^{i \theta}\right)$ is situated in (or near) the two lobes of dominance $\mathbf{L}^{ \pm}$given by $\left|1 \pm i \eta^{2} /(4 \pi)\right| \leq 1$, where the contributions $E_{k}^{ \pm}(\eta)$ become exponentially large. These lobes correspond to the portions of the lemniscates $|\eta|^{2}= \pm 8 \pi \sin (2 \theta)$ lying in $\operatorname{Re}(\eta)<0$; see Figure 2 and $\S 6$ for a discussion of the choice of branch cuts in the $\eta$ plane.

\section{Numerical results and discussion}

In the following discussion of the coefficients in the expansion of $Q(a, z)=\Gamma(a, z) / \Gamma(a)$, we take $|\arg (a)| \leq \pi$ and restrict our attention to values of $z$ in the sector $|\arg (z)| \leq \pi$. Values of $\arg (z)$ outside this range are covered by the analytic continuation formula $[8$, p. 45]

$$
Q\left(a, z e^{2 \pi m i}\right)=e^{2 \pi m i a}\{Q(a, z)-1\}+1 \quad(m=0, \pm 1, \pm 2, \ldots) .
$$

It then follows that the parameter $\lambda=z / a$ satisfies $|\arg (\lambda)| \leq 2 \pi$.

The connection between the variables $\eta$ and $\lambda$ (or $\mu$ ) is specified by (1.2) and (1.3). Figure 3 depicts the $\lambda-\eta$ mapping, from the sheet $-2 \pi \leq \arg (\lambda) \leq 2 \pi$ to the $\eta$ plane, with various corresponding points labeled from $A$ to $F$ and $D^{\prime}$ to $F^{\prime}$. There are branch points in this mapping at the points $\lambda=e^{ \pm 2 \pi i}$, which correspond to $\eta=2 \sqrt{ } \pi e^{ \pm \frac{3}{4} \pi i}$ (labeled $G$ and $G^{\prime}$, respectively, in the figures). The curves $D B G E$ and $E^{\prime} G^{\prime} B D^{\prime}$ in Figure $3(\mathrm{a}),(\mathrm{b})$, which correspond to the rays $|\arg (\eta)|=\frac{1}{4} \pi$ and $\frac{3}{4} \pi$ in the $\eta$ plane, are given by

$$
\operatorname{Im} \lambda= \pm\left\{\exp [2(\operatorname{Re} \lambda-1)]-(\operatorname{Re} \lambda)^{2}\right\}^{\frac{1}{2}} .
$$

When $a>0$, these curves are the boundaries of the regions in which $Q(a, z)$ is exponentially large or small; compare (1.1).

The details of the correspondence between $\eta$ and $\lambda$ are shown in Figure 4. This represents points in the $\eta$ plane corresponding to given $\lambda=\rho e^{i \phi}$ : the curves show the paths in the $\eta$ plane for fixed $\rho$ and $\phi$. The figure for $-2 \pi \leq \arg (\lambda) \leq 2 \pi$ is symmetrical with respect to the Re $\eta$ axis. The branch cuts in the upper half-plane from the branch points $\eta=2 \sqrt{ } \pi e^{ \pm \frac{3}{4} \pi i}$ are chosen to correspond to the curves with $\phi= \pm 2 \pi$, respectively; cf. also Figure 2 . 


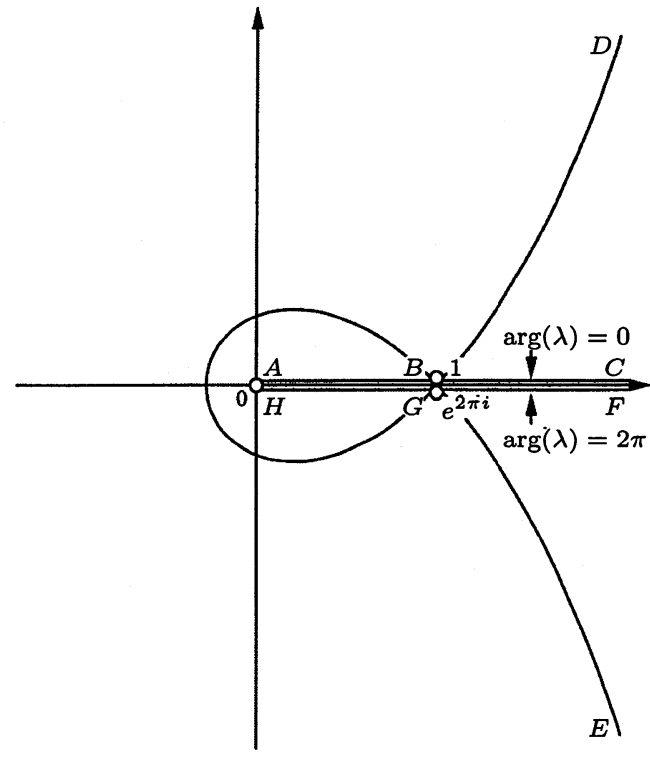

(a)

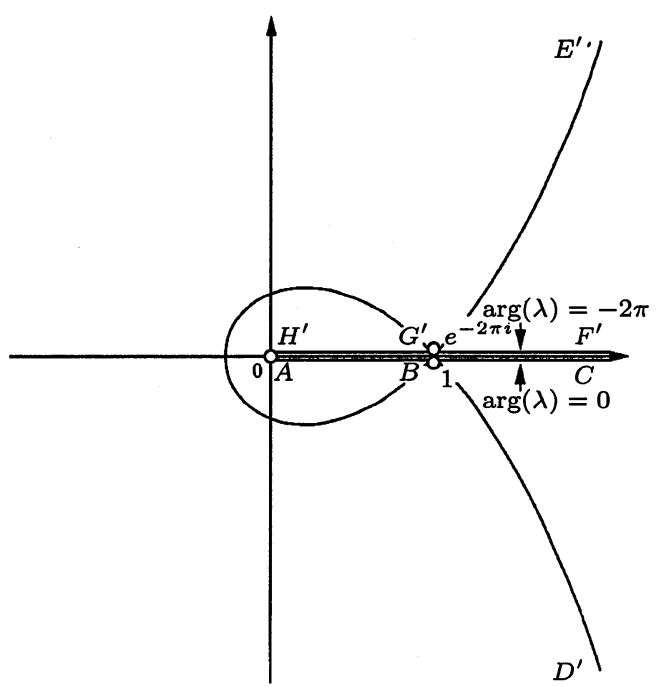

(b)

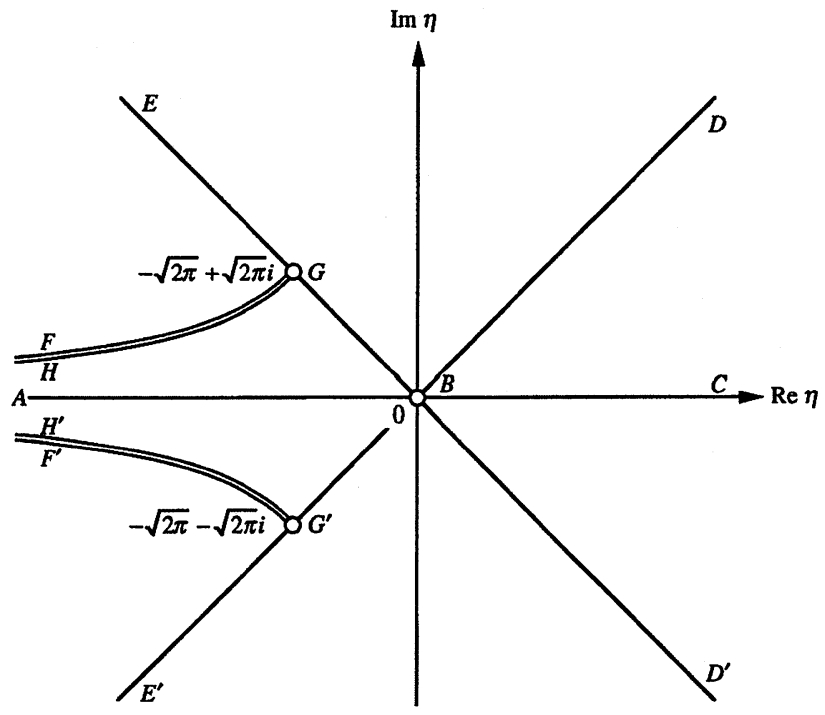

(c)

Figure 3. The $\lambda-\eta$ mapping: (a) the $\lambda$ plane $(0 \leq \arg (\lambda) \leq 2 \pi)$, (b) the $\lambda$ plane $(-2 \pi \leq \arg (\lambda) \leq 0)$, and (c) the $\eta$ plane. 


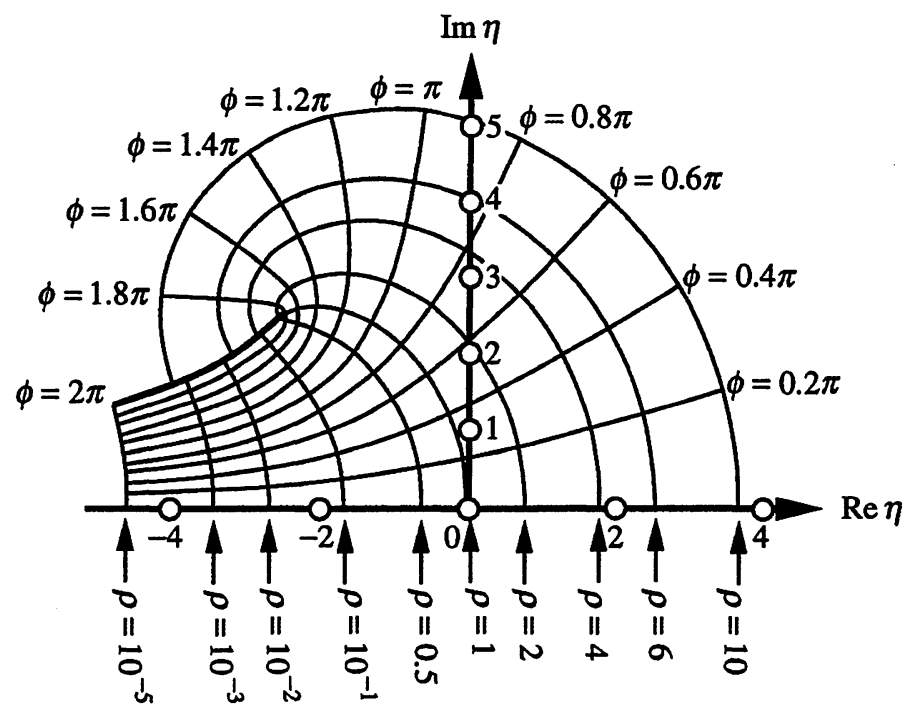

FIGURE 4. The details of the $\lambda-\eta$ mapping: the upper half $\eta$ plane for different $\rho$ and $\phi$ where $\lambda=\rho e^{i \phi}$. The dark curve $\phi=2 \pi$ corresponds to the upper branch cut.

The coefficients $c_{k}(\eta)$ for a given value of $\eta$ were computed numerically from (1.4) and (1.5) with $\mu(=\lambda-1)$ determined from (1.2) (see [13]); the factor $f_{k}(\eta)$ is then obtained from (5.6). For small values of $\eta$, it is found more convenient to determine $c_{k}(\eta)$ from the power series (5.1). In Figure 5, we present the behavior of $f_{k}(\eta)$ for real values of $\eta$ (i.e., $\lambda>0$ ) for different values of $k$. The asymptotic values predicted by (5.13), (5.14) (or (4.3)) cannot be distinguished from the numerically computed values on this scale. It is seen that $f_{k}(\eta)$ decreases smoothly for $\eta>0$, while for $\eta<0$, there is an oscillatory zone in which $f_{k}(\eta)$ "feels" the presence of the nearby lobes $\mathbf{L}^{ \pm}$ before settling down to a constant limiting value as $\eta \rightarrow-\infty$. Noting that $\mu \sim \frac{1}{2} \eta^{2}$ as $\eta \rightarrow+\infty$ and $\mu \sim-1$ as $\eta \rightarrow-\infty$, we obtain for large $k$ from (5.13) and (5.14), or from (3.13) and (4.2), the limits as $\eta \rightarrow \pm \infty$

$\lim _{\eta \rightarrow+\infty} \eta^{2} f_{k}(\eta)=\left\{\begin{array}{l}(-)^{(k+1) / 2} 4 k^{-\frac{1}{2}} \\ (-)^{k / 2} \frac{2}{3} \pi k^{-\frac{3}{2}}\end{array} \quad, \quad \lim _{\eta \rightarrow-\infty} f_{k}(\eta)=\left\{\begin{array}{ll}(-)^{(k-1) / 2} 2 k^{-\frac{1}{2}} & (k \text { odd }) \\ (-)^{k / 2+1} \frac{1}{3} \pi k^{-\frac{3}{2}} & (k \text { even })\end{array}\right.\right.$.

This leading behavior corresponds to the result $c_{k}(\eta) \sim \gamma_{k} / \mu$ as $\eta \rightarrow \pm \infty$ given in [13, p. 762]. The oscillatory zone in Figure 5 can be accounted for by writing the leading asymptotic terms of $f_{k}(\eta)$ in (5.13) and (5.14) (or (3.13) and (4.2)) in the form

$$
f_{k}(\eta) \sim 2[M(\eta)]^{-\kappa} \cos \left\{\left(\frac{1}{2} \pi-\phi\right) \kappa\right\}+ \begin{cases}(-)^{(k+1) / 2} 2 /\left(\mu k^{\frac{1}{2}}\right) & (k \text { odd }) \\ (-)^{k / 2} 4 \pi Q_{1}(\mu) /\left(\mu^{3} k^{\frac{3}{2}}\right) & (k \text { even })\end{cases}
$$

for $k \rightarrow \infty$ in $\eta<0$ where $M(\eta)=\left\{1+\left[\eta^{2} /(4 \pi)\right]^{2}\right\}^{\frac{1}{2}}$ and $\phi=\arg \left\{1+i \eta^{2} /(4 \pi)\right\}$. Since, for $\eta<0, M(\eta)>1$ and $0<\phi<\frac{1}{2} \pi$, it follows that as $k$ increases, the 


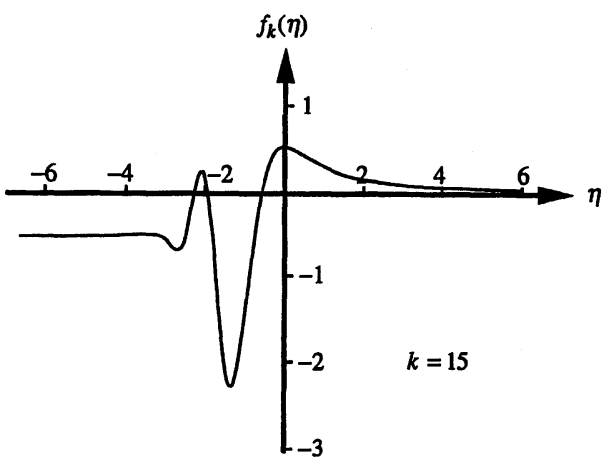

(a)

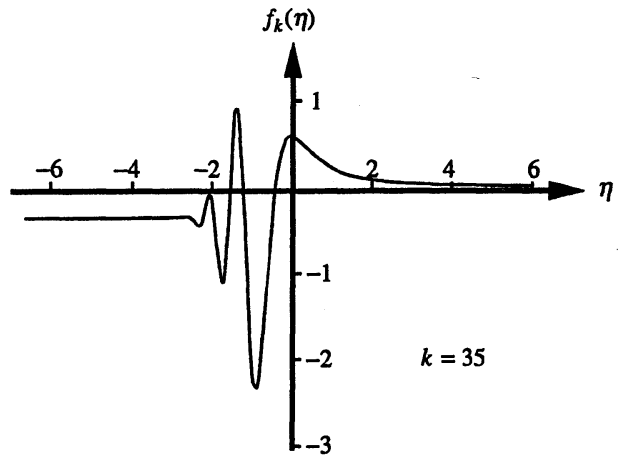

(c)

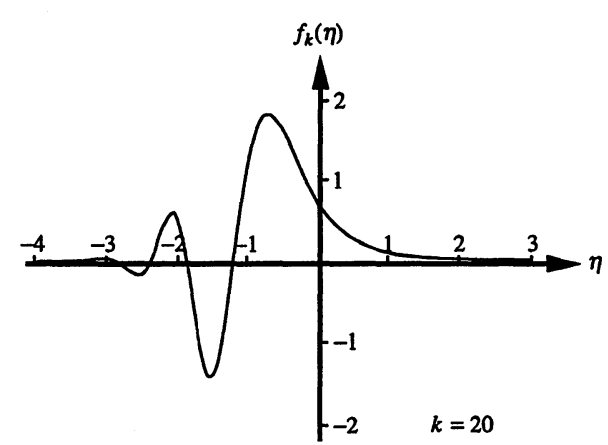

(b)

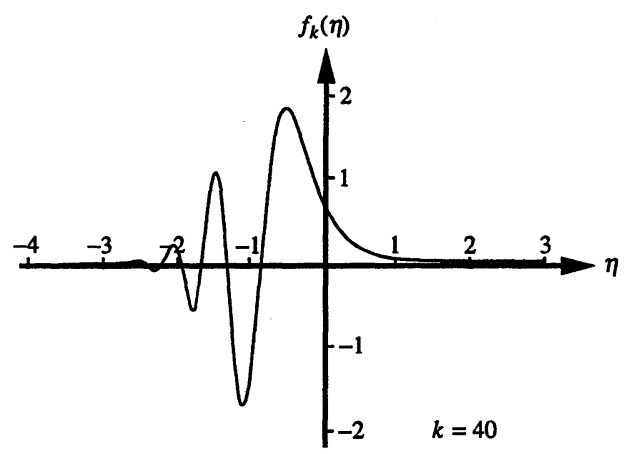

(d)

FIGURE 5. The behavior of $f_{k}(\eta)$ for real $\eta$ and different values of $k$. The asymptotic and exact behaviors are indistinguishable on this scale.

oscillatory zone and the wavelength of the oscillations will decrease. This tendency is clearly visible in Figure 5.

In Table 1, we give the computed values of $\left|f_{k}(\eta)\right|$ compared with the leading asymptotic behavior for different $|\eta|$ and varying $\theta=\arg (\eta)$ for $0 \leq \theta \leq \pi$. It is seen that as one enters the upper lobe $\mathbf{L}^{+}$(the behavior in the lower lobe $\mathbf{L}^{-}$is given by the conjugate value), $\left|f_{k}(\eta)\right|$ increases dramatically; this behavior is illustrated in Figure 6.

One consequence of this rapid growth of $\left|f_{k}(\eta)\right|$ in the lobes (or in the corresponding domains in the $\lambda$ plane) is that the behavior of the high-order terms in the expansion (1.1) will no longer be controlled by the simple "factorial divided by a power" dependence when $a$ and $z$ are such that $\eta$ lies in either $\mathbf{L}^{ \pm}$. This change in the growth of 


\begin{tabular}{|c|c|c|c|c|}
\hline \multirow[b]{2}{*}{$\theta / \pi$} & \multicolumn{2}{|c|}{$\eta=0.5 \exp (i \theta)$} & \multicolumn{2}{|c|}{$\eta=1.0 \exp (i \theta)$} \\
\hline & $\left|f_{k}(\eta)\right|$ & Asymptotic & $\left|f_{k}(\eta)\right|$ & Asymptotic \\
\hline 0 & $0.43379(0)$ & $0.43542(0)$ & $0.29519(0)$ & $0.29658(0)$ \\
\hline 0.20 & $0.47734(0)$ & $0.47901(0)$ & $0.34690(0)$ & 0.34829( \\
\hline 0.40 & 0.62063 & $0.62245(0)$ & $0.62519(0)$ & $0.62664(0)$ \\
\hline 0.60 & 0.80016( & $0.80215(0)$ & $0.19236(1)$ & $0.19257(1)$ \\
\hline 0.70 & 0.78798( & $0.78990(0)$ & $0.28770(1)$ & 0.28789( \\
\hline 0.75 & $0.72945(0)$ & $0.73126(0)$ & $0.30039(1)$ & $0.30051(1)$ \\
\hline 0.80 & 0.63457( & $0.63623(0)$ & $0.27851(1)$ & $0.27852(1)$ \\
\hline 1.00 & 0.12974 & $0.13207(0)$ & $0.14791(1)$ & $0.14764(1)$ \\
\hline & \multicolumn{2}{|c|}{$\eta=2.0 \exp (i \theta)$} & \multicolumn{2}{|c|}{$\eta=3.0 \exp (i \theta)$} \\
\hline$\theta / \pi$ & $\left|f_{k}(\eta)\right|$ & Asymptotic & $\left|f_{k}(\eta)\right|$ & Asymptotic \\
\hline 0 & $0.14078(0)$ & $0.14184(0)$ & $0.78555(-1)$ & $0.79413(-1)$ \\
\hline 0.20 & 0.16013( & 0.16109 & $0.85673(-1)$ & $0.86413(-1)$ \\
\hline 0.40 & 0.23373( & 0.23399( & $0.10446(0)$ & 0.10480( \\
\hline 0.60 & 0.11876 & 0.11873 & $0.21941(2)$ & 0.21940 \\
\hline 0.70 & 0.22964 & 0.22965 & $0.23482(7)$ & 0.23482 \\
\hline 0.75 & 0.37930 & 0.37930 & 0.30078 ( 9) & 0.30078 \\
\hline 0.80 & 0.23006( & 0.23006 & $0.23482(7)$ & 0.23482 \\
\hline 1.00 & $0.16796(0)$ & 0.17147( & $0.56611(0)$ & 0.56276 \\
\hline \multirow[b]{2}{*}{$\theta / \pi$} & \multicolumn{2}{|c|}{$\eta=4.0 \exp (i \theta)$} & \multicolumn{2}{|c|}{$\eta=6.0 \exp (i \theta)$} \\
\hline & $\left|f_{k}(\eta)\right|$ & Asymptotic & $\left|f_{k}(\eta)\right|$ & Asymptotic \\
\hline 0 & $0.49522(-1)$ & $0.50239(-1)$ & $0.24616(-1)$ & $0.25200(-1)$ \\
\hline 0.20 & $0.53017(-1)$ & $0.53622(-1)$ & $0.25862(-1)$ & $0.26339(-1)$ \\
\hline 0.40 & $0.64529(-1)$ & $0.64770(-1)$ & $0.29913(-1)$ & $0.30062(-1)$ \\
\hline 0.60 & $0.33765(0)$ & $0.33850(0)$ & $0.37618(-1)$ & $0.37138(-1)$ \\
\hline 0.70 & 0.26849 & $0.26849(6)$ & $0.40358(-1)$ & $0.39425(-1)$ \\
\hline 0.75 & 0.54147 & 0.54147 & $0.39324(-1)$ & $0.38274(-1)$ \\
\hline 0.80 & 0.26849 & 0.26849 & $0.36286(-1)$ & $0.35295(-1)$ \\
\hline $1.00^{*}$ & 0.52110 & $0.51794(0)$ & $0.52032(0)$ & $0.51707(0)$ \\
\hline
\end{tabular}

TABLE 1. The numerical values of $\left|f_{k}(\eta)\right|$ (the numbers in brackets denote a multiplicative power of 10) compared with the leading asymptotic approximation when $k=15$ for varying $\theta=\arg (\eta)$ and different $|\eta|$. The asterisk signifies the value of $\eta$ below the branch cut.

the coefficients $c_{k}(\eta)$ clearly has implications for optimal truncation, and hence the accuracy directly attainable from (1.1) as the phases of $a$ and $z$ vary at, for example, fixed $|a|$ and $|z|$. We also remark that essentially the same coefficients appear in an asymptotic expansion for the generalized exponential integral (or, equivalently, the incomplete gamma function with negative values of the parameter). Hence, the behavior of the coefficients, demonstrated in the present study, also has implications in explicit error bounds in an expansion, up to $n$ terms, recently obtained by Dunster [7]: these error bounds explicitly involve the $n$th coefficient of the expansion (see [7, Eq. (3.67) and Theorems 6.1 and 6.2]). 


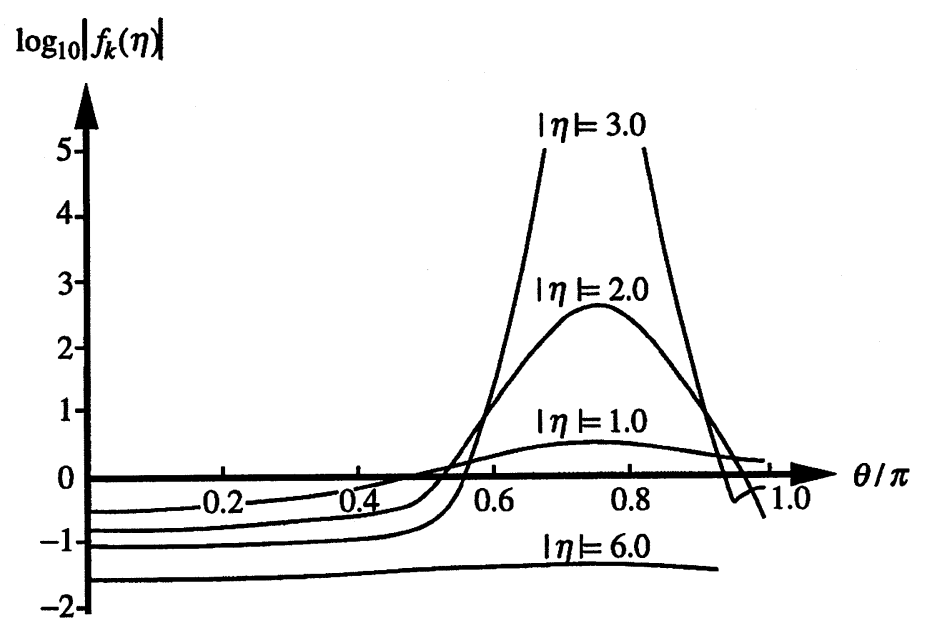

FIGURE 6. The behavior of $\left|f_{k}(\eta)\right|$ as a function of $\theta=\arg (\eta)$ with fixed $|\eta|$ when $k=15$.

At the transition point of $\Gamma(a, z)$, which corresponds to $z=a$ (i.e., at $\mu=0, \eta=0$ ), we have, from (2:11) and (3.12), $u_{\lambda}^{ \pm}=0$ and $\mathcal{A}_{m}^{ \pm}(0)=0$, so that the coefficients $a_{m}^{ \pm}(0)$ in (3.15) consist of a sum involving the coefficients $f_{j}(0), j=0,1, \ldots, m$. From (3.14), we accordingly obtain the result

$$
f_{k}(0) \sim(-)^{k} \sin \left(\frac{1}{2} \pi \kappa\right)+\frac{1}{\pi} \sum_{m=0}^{\infty} \frac{\Gamma\left(m+\frac{1}{2}\right)}{\kappa^{m+\frac{1}{2}}} d_{m}(k), \quad k \rightarrow \infty
$$

where

$$
d_{m}(k)=\sum_{j=0}^{m} \sin \left(\frac{1}{2} \pi(k-j)\right) b_{m-j}^{(m)} f_{j}(0) .
$$

Evaluation of the first two coefficients $d_{m}(k)$ leads to the approximation

$$
f_{k}(0) \sim(-)^{k} \sin \left(\frac{1}{2} \pi \kappa\right)+\frac{2}{3 \sqrt{ } \kappa}\left(1+\frac{3}{8 \kappa}\right) \sin \left(\frac{1}{2} \pi k\right)+\frac{\pi}{135 \kappa^{\frac{3}{2}}} \cos \left(\frac{1}{2} \pi k\right),
$$

which agrees with the (less precise) result in (5.15). It is worth pointing out that the leading term of the above approximation is also in agreement with that given by Dingle [5, p. 162], who obtained an expansion of the large-order coefficients in the incomplete gamma function ${ }^{5}[a, z]$ ! at the point $z=a$.

Finally, we wish to remark that the expansion of the high-order coefficients $c_{k}(\eta)$ $(k \gg 1)$ in (3.13)-(3.15) involves terms which depend on the low-order coefficients $c_{0}(\eta), c_{1}(\eta), \ldots$. This is an example of the resurgence-like property exhibited by coefficients in an asymptotic expansion.

\footnotetext{
${ }^{5}$ The incomplete gamma function in [5] is denoted by $[a, z] !$ where $[a, z] !=\Gamma(a+1, z)=a \Gamma(a, z)+$ $z^{a} e^{-z}$.
} 
Acknowledgment. We thank the referees for a number of helpful comments. Author T. M. D. would like to thank the Division of Mathematical Sciences at the University of Abertay Dundee for its hospitality during the course of this investigation and is pleased to acknowledge the financial support of the National Science Foundation under Grant DMS 9404389. Author S. C. wishes to acknowledge the financial support of a Research Studentship at the University of Abertay Dundee.

\section{Appendix A. An expression for the partial sums $c_{k}^{(r)}(\eta)$}

In this appendix, we establish the relation between the partial sums $c_{k}^{(r)}(\eta)$ defined in (5.4) and the coefficients $c_{j}(\eta)(j=0,1, \ldots, k-r)$ given by

$$
c_{k}^{(r)}(\eta)=\gamma_{r}\left\{\sum_{j=0}^{k-r}(-)^{j} \gamma_{j} c_{k-r-j}(\eta)+\delta_{k r}\right\} \quad(r \leq k)
$$

where $\delta_{k r}$ denotes the Kronecker delta symbol.

From (5.3), the series expansion of $\mu-\eta$ is

$$
\mu-\eta=\sum_{n=2}^{\infty} \alpha_{n} \eta^{n} \quad(|\eta|<2 \sqrt{ } \pi)
$$

Repeated application of the differential operator $\eta^{-1} d / d \eta$ yields, for $m=1,2, \ldots$,

$$
\begin{aligned}
\left(\frac{1}{\eta} \frac{d}{d \eta}\right)^{m}(\mu-\eta) & =2^{m} \sum_{n=-2 m+2}^{\infty} \alpha_{n+2 m}\left(\frac{1}{2} n+1\right)_{m} \eta^{n} \\
& =2^{m} \sum_{n=0}^{\infty} \alpha_{n+2 m}\left(\frac{1}{2} n+1\right)_{m} \eta^{n}+R_{m}
\end{aligned}
$$

where

$$
R_{m}=2^{m} \sum_{n=-2 m+3}^{-1} \alpha_{n+2 m}\left(\frac{1}{2} n+1\right)_{m} \eta^{n} \quad(m \geq 2),
$$

$(a)_{m}=\Gamma(a+m) / \Gamma(a)$, and the prime denotes summation over odd values of $n$. Replacement of the summation index $n$ by $-2 r-1$ then shows that

$$
R_{m}=2^{m} \sum_{j=0}^{m-2} \frac{\alpha_{2 m-2 j-1}}{\eta^{2 j+1}}\left(\frac{1}{2}-j\right)_{m}=(-)^{m-1} \sum_{j=0}^{m-2} 2^{j}\left(\frac{1}{2}\right)_{j} \frac{\gamma_{m-j-1}}{\eta^{2 j+1}} \quad(m \geq 2)
$$

where we have used the result relating the odd-order coefficients $\alpha_{2 k+1}$ to the Stirling coefficients given by [13]

$$
\alpha_{2 k+1}=\frac{(-)^{k} \gamma_{k} \Gamma\left(\frac{1}{2}\right)}{2^{k+1} \Gamma\left(k+\frac{3}{2}\right)} .
$$

From the definition of $c_{k}(\eta)$ in (1.4) and, from (1.2), the fact that $d \mu / d \eta=\eta(1+$ $\mu) / \mu$, we obtain successively

$$
\begin{gathered}
\frac{1}{\eta} \frac{d}{d \eta}(\mu-\eta)=1+c_{0}(\eta) \\
\left(\frac{1}{\eta} \frac{d}{d \eta}\right)^{2}(\mu-\eta)=c_{1}(\eta)-\frac{\gamma_{1}}{\mu}=\gamma_{0} c_{1}(\eta)-\gamma_{1} c_{0}(\eta)-\frac{\gamma_{1}}{\eta}
\end{gathered}
$$


and the general form, for $m \geq 2$,

$$
\left(\frac{1}{\eta} \frac{d}{d \eta}\right)^{m}(\mu-\eta)=\sum_{j=0}^{m-1}(-)^{j} \gamma_{j} c_{m-j-1}(\eta)+R_{m}
$$

where we recall from $\S 1$ that $\gamma_{0}=1$. The result (A.5) can be established by induction on $m$ as follows. Using (1.4), we have

$$
\begin{aligned}
\left(\frac{1}{\eta} \frac{d}{d \eta}\right)^{m+1}(\mu-\eta) & =\sum_{j=0}^{m-1}(-)^{j} \gamma_{j}\left(c_{m-j}(\eta)-\frac{\gamma_{m-j}}{\mu}\right)+\frac{1}{\eta} \frac{d}{d \eta} R_{m} \\
& =\sum_{j=0}^{m}(-)^{j} \gamma_{j}\left(c_{m-j}(\eta)-\frac{\gamma_{m-j}}{\mu}\right)+(-)^{m} \frac{\gamma_{m}}{\eta}+\frac{1}{\eta} \frac{d}{d \eta} R_{m} \\
& =\sum_{j=0}^{m}(-)^{j} \gamma_{j} c_{m-j}(\eta)+R_{m+1}
\end{aligned}
$$

since the finite sum $R_{m}$ in (A.3) satisfies the recursion

$$
R_{m+1}=(-)^{m} \frac{\gamma_{m}}{\eta}+\frac{1}{\eta} \frac{d}{d \eta} R_{m} \quad(m \geq 2)
$$

and $^{6}$

$$
\sum_{j=0}^{m}(-)^{j} \gamma_{j} \gamma_{m-j}=0
$$

Hence, (A.5) holds for $m \geq 2$ by induction.

Comparison of (A.2) with (A.5) together with use of (A.4) when $m=1$ then shows that

$$
2^{m} \sum_{n=0}^{\infty} \alpha_{n+2 m}\left(\frac{1}{2} n+1\right)_{m} \eta^{n}=\sum_{j=0}^{m-1}(-)^{j} \gamma_{j} c_{m-j-1}(\eta)+\delta_{m 1} \quad(m \geq 1)
$$

whence, from the definition of the partial sums $c_{k}^{(r)}(\eta)$ in (5.4) and identification of $m$ with $k-r+1$, the result (A.1) follows. We remark that although (A.1) has been established in $|\eta|<2 \sqrt{ } \pi$, this restriction can be removed by appeal to analytic continuation.

\section{Appendix B. The large $k$ behavior of $f_{k}^{(0)}(\eta)$}

We determine the behavior as $k \rightarrow \infty$ of $f_{k}^{(0)}(\eta)$ defined in (5.8) by

$$
f_{k}^{(0)}(\eta)=\frac{(-4 \pi)^{k+1}}{\Gamma(\kappa)} \sum_{n=0}^{\infty} \alpha_{n+2 k+2} \frac{\Gamma\left(\frac{1}{2} n+k+2\right)}{\Gamma\left(\frac{1}{2} n+1\right)} \eta^{n} \quad(|\eta|<2 \sqrt{ } \pi)
$$

${ }^{6}$ This follows from the asymptotic expansions of $\Gamma(z)$ and $1 / \Gamma(z)$ which involve the sums $\sum(-)^{j} \gamma_{j} z^{-j}$ and $\sum \gamma_{j} z^{-j}$, respectively. The result (A.7) is the statement that the coefficients in the product of these expansions must vanish for $j \geq 1$. For odd $m$, we note that this result is a simple identity. 
where the coefficients $\alpha_{k}$ are defined by (5.3). From the expansion [4]

$$
\begin{aligned}
\alpha_{m}=\frac{(-)^{m}}{(2 \sqrt{ } \pi)^{m}} \frac{\Gamma\left(\frac{1}{2} m-\frac{1}{2}\right)}{\Gamma\left(\frac{1}{2} m+1\right)}\left\{-\sin \left\{\left(\frac{m-1}{4}\right) \pi\right\}\right. \\
\left.\quad+\frac{\pi}{3 m} \cos \left\{\left(\frac{m-1}{4}\right) \pi\right\}+O\left(m^{-2}\right)\right\}
\end{aligned}
$$

for $m \rightarrow \infty$, we can approximate the coefficients $\alpha_{n+2 k+2}$ in (B.1) uniformly in $n$ as $k \rightarrow \infty$. We therefore find, with $\Psi_{n}=\frac{1}{2} \pi\left(\frac{1}{2} n+\kappa\right)$,

$$
\begin{aligned}
f_{k}^{(0)}(\eta)= & \frac{(-)^{k}}{\Gamma(\kappa)} \sum_{n=0}^{\infty}\left(-\frac{\eta}{2 \sqrt{ } \pi}\right)^{n} \frac{\Gamma\left(\frac{1}{2} n+\kappa\right)}{\Gamma\left(\frac{1}{2} n+1\right)} \\
& \quad \times\left\{\sin \left(\Psi_{n}\right)-\frac{\pi}{6\left(\frac{1}{2} n+k+1\right)} \cos \left(\Psi_{n}\right)+O\left(k^{-2}\right)\right\} \\
= & A_{k}-\frac{\pi}{6 k} B_{k}+O\left(k^{-2}\right)
\end{aligned}
$$

where

$$
\begin{aligned}
& A_{k}=\frac{(-)^{k}}{2 i}\left[e^{\frac{1}{2} \pi i \kappa} F_{k}\left(e^{\frac{1}{4} \pi i} X\right)-e^{-\frac{1}{2} \pi i \kappa} F_{k}\left(e^{-\frac{1}{4} \pi i} X\right)\right] \\
& B_{k}=\frac{(-)^{k}}{2}\left[e^{\frac{1}{2} \pi i \kappa} F_{k-1}\left(e^{\frac{1}{4} \pi i} X\right)+e^{-\frac{1}{2} \pi i \kappa} F_{k-1}\left(e^{-\frac{1}{4} \pi i} X\right)\right]
\end{aligned}
$$

$X=\eta /(2 \sqrt{ } \pi)$, and we have defined the function $F_{k}(z)$ by

$$
F_{k}(z)=\frac{1}{\Gamma(\kappa)} \sum_{n=0}^{\infty}(-z)^{n} \frac{\Gamma\left(\frac{1}{2} n+\kappa\right)}{\Gamma\left(\frac{1}{2} n+1\right)} \quad(|z|<1) .
$$

In Appendix $\mathrm{C}$, it is shown that $F_{k}(z)$ can be expressed in terms of a hypergeometric function by

$$
F_{k}(z)=\left\{\begin{array}{ll}
\frac{k !}{\sqrt{ } \pi \Gamma(\kappa+1)}{ }_{2} F_{1}\left(\frac{1}{2}, \kappa ; \kappa+1 ; 1-z^{2}\right) & \left(|\arg (z)| \leq \frac{1}{2} \pi\right) \\
2\left(1-z^{2}\right)^{-\kappa}-F_{k}(-z) & \left(\frac{1}{2} \pi<|\arg (z)| \leq \pi\right)
\end{array} .\right.
$$

This result supplies the analytic continuation of the sum on the right-hand side of (B.5), thereby enabling the computation of $F_{k}(z)$ in $|z|>1$. A uniform asymptotic approximation to $F_{k}(z)$ for $k \rightarrow \infty$ valid in $|\arg (z)| \leq \frac{1}{2} \pi$ (and unrestricted finite $|z|$ ) is given in terms of a complementary error function in (C.7). When $|z|$ is bounded away from zero, this leads to the behavior given by (see (C.8) and (C.9))

$$
F_{k}(z)= \begin{cases}\frac{1}{z \sqrt{\pi k}}\left\{1+\frac{1}{2 k}\left(\frac{1}{4}-\frac{1}{z^{2}}\right)+O\left(k^{-2}\right)\right\} & \left(|\arg (z)| \leq \frac{1}{2} \pi\right) \\ 2\left(1-z^{2}\right)^{-\kappa}+\frac{1}{z \sqrt{\pi k}}\left\{1+\left(\frac{1}{4}-\frac{1}{z^{2}}\right)+O\left(k^{-2}\right)\right\} & \left(\frac{1}{2} \pi<|\arg (z)| \leq \pi\right)\end{cases}
$$

as $k \rightarrow \infty$. It thus is seen that, in the adjacent sectors $\frac{1}{2} \pi<|\arg (z)| \leq \pi$, the behavior of $F_{k}(z)$ contains the term $2\left(1-z^{2}\right)^{-\kappa}$. As $k \rightarrow \infty$, this latter term will dominate the behavior of $F_{k}(z)$ when $z\left(=r e^{i \theta}\right)$ lies in the domain bounded by $\left|1-z^{2}\right|=1$; that is, in the domain bounded by the left-hand half of the lemniscate $r^{2}=2 \cos (2 \theta)$, with the plane cut along the negative $z$ axis between -1 and $-\infty$. 
Substitution of (B.7) into (B.3) and (B.4) then shows that when $|\eta|$ is bounded away from zero (but otherwise unrestricted),

$$
f_{k}^{(0)}(\eta)= \begin{cases}D_{k}(\eta) & \left(|\arg (\eta)| \leq \frac{1}{2} \pi\right) \\ D_{k}(\eta)+E_{k}^{+}(\eta)+E_{k}^{-}(\eta) & \left(\frac{1}{2} \pi<|\arg (\eta)| \leq \pi\right)\end{cases}
$$

as $k \rightarrow \infty$, where

$$
\begin{gathered}
D_{k}(\eta)=\frac{2(-)^{k}}{\eta \sqrt{ } k}\left\{\left(1+\frac{3}{8 k}\right) \sin \left(\frac{1}{2} \pi k\right)-\frac{\pi}{6 k}\left(1-\frac{12}{\eta^{2}}\right) \cos \left(\frac{1}{2} \pi k\right)+O\left(k^{-2}\right)\right\} \\
E_{k}^{ \pm}(\eta)=e^{ \pm \frac{1}{2} \pi i \kappa}\left(1 \pm \frac{i \eta^{2}}{4 \pi}\right)^{-\kappa}\left\{1+O\left(k^{-1}\right)\right\}
\end{gathered}
$$

\section{Appendix C. The function $F_{k}(z)$}

The function $F_{k}(z)$ is defined by

$$
F_{k}(z)=\frac{1}{\Gamma(\kappa)} \sum_{n=0}^{\infty}(-z)^{n} \frac{\Gamma\left(\frac{1}{2} n+\kappa\right)}{\Gamma\left(\frac{1}{2} n+1\right)}
$$

in the unit disc $|z|<1$. By separating the right-hand side of (C.1) into sums over even and odd values of $n$, we can express $F_{k}(z)$ in terms of the Gauss hypergeometric function as

$$
\begin{aligned}
F_{k}(z) & =\left(1-z^{2}\right)^{-\kappa}-\frac{2 z k !}{\sqrt{ } \pi \Gamma(\kappa)}{ }_{2} F_{1}\left(1, k+1 ; \frac{3}{2} ; z^{2}\right) \\
& =\frac{k !}{\sqrt{ } \pi \Gamma(\kappa+1)}{ }_{2} F_{1}\left(\frac{1}{2}, \kappa ; \kappa+1 ; 1-z^{2}\right) \quad\left(|\arg (z)| \leq \frac{1}{2} \pi\right) .
\end{aligned}
$$

upon making use of [12, p. 34, Eq. (1.8.10)].

From (C.2), we then obtain

$$
F_{k}(z)=2\left(1-z^{2}\right)^{-\kappa}-F_{k}(-z),
$$

which enables the computation of $F_{k}(z)$ in the sectors $\frac{1}{2} \pi<|\arg (z)| \leq \pi$. The result (C.3) combined with (C.4) indicates that $F_{k}(z)$ has a branch point at $z=-1$ and supplies its analytic continuation into the domain $|z|>1$.

An asymptotic approximation for $F_{k}(z)$ as $k \rightarrow \infty$ can be obtained by using the Euler integral representation for the hypergeometric function in (C.3) $[1$, p. 558] to find, when ${ }^{7}|\arg (z)| \leq \frac{1}{2} \pi$,

$$
\begin{aligned}
F_{k}(z) & =\frac{k !}{\sqrt{ } \pi \Gamma(\kappa)} \int_{0}^{1} t^{k-\frac{1}{2}}\left[1-\left(1-z^{2}\right) t\right]^{-\frac{1}{2}} d t \\
& =\frac{k !}{\sqrt{ } \pi \Gamma(\kappa)}\left(1-z^{2}\right)^{-\frac{1}{2}} \int_{0}^{\infty} e^{-\kappa u}\left[\chi-\left(e^{-u}-1\right)\right]^{-\frac{1}{2}} d u
\end{aligned}
$$

\footnotetext{
${ }^{7}$ The $z^{2}$ plane is cut along the negative real axis in (C.5). As the first parameter in the hypergeometric function in (C.3) equals $\frac{1}{2}$, the region of validity of the integral can be continued onto the cut $\left[8\right.$, p. 161], with the result that (C.5) holds for $|\arg (z)| \leq \frac{1}{2} \pi$.
} 
where $\kappa=k+\frac{1}{2}$ and $\chi=z^{2} /\left(1-z^{2}\right)$. For large $k$, the dominant contribution to this integral arises from the neighborhood of $u=0$. Expanding the slowly varying factor $\left[\chi-\left(e^{-u}-1\right)\right]^{-\frac{1}{2}}$ for small $u$, we therefore have

$$
\begin{aligned}
F_{k}(z) & =\frac{k !}{\sqrt{ } \pi \Gamma(\kappa)}\left(1-z^{2}\right)^{-\frac{1}{2}} \int_{0}^{\infty} e^{-\kappa u}(\chi+u)^{-\frac{1}{2}}\left\{1+\frac{u^{2}}{4(\chi+u)}+\cdots\right\} d u \\
& =\frac{k !}{\sqrt{\pi \kappa} \Gamma(\kappa)}\left(1-z^{2}\right)^{-\frac{1}{2}}\left\{C_{0}+\frac{C_{1}}{\kappa}+\cdots\right\}, \quad k \rightarrow \infty,
\end{aligned}
$$

where use has been made of the integral

$$
\int_{0}^{\infty} e^{-\kappa u}(\chi+u)^{-p} d u=\kappa^{p-1} e^{\kappa \chi} \Gamma(1-p, \kappa \chi),
$$

together with recursive properties of the incomplete gamma function, to show that

$$
\begin{gathered}
C_{0}=e^{\kappa \chi} \Gamma\left(\frac{1}{2}, \kappa \chi\right), \\
C_{1}=\frac{1}{2} e^{\kappa \chi} \Gamma\left(\frac{1}{2}, \kappa \chi\right)\left(1-4 \kappa \chi-4 \kappa^{2} \chi^{2}\right)+(\kappa \chi)^{\frac{1}{2}}(1+2 \kappa \chi) .
\end{gathered}
$$

We note that $C_{1} \sim 2(\kappa \chi)^{-\frac{3}{2}}$ for large $k$ when $|\chi| \neq 0$, so that the order of the second term in braces in (C.6) changes smoothly from $\mathrm{O}\left(\kappa^{-1}\right)$ when $\chi=0$ to $\mathrm{O}\left(\kappa^{-\frac{5}{2}}\right)$ when $|\chi|$ is bounded away from zero.

Since $\Gamma\left(\frac{1}{2}, z^{2}\right)=\sqrt{ } \pi \operatorname{erfc}(z)$, the approximate form of $F_{k}(z)$ as $k \rightarrow \infty$, uniformly valid in $z$ for $|\arg (z)| \leq \frac{1}{2} \pi$, is therefore

$$
F_{k}(z) \sim \frac{k !}{\sqrt{ } \kappa \Gamma(\kappa)}\left(1-z^{2}\right)^{-\frac{1}{2}} e^{\kappa \chi} \operatorname{erfc}(\sqrt{\kappa \chi}), \quad k \rightarrow \infty
$$

For $|z|$ bounded away from zero, we then obtain from the asymptotic expansion of the complementary error function ${ }^{8}$ and the fact that $C_{1}=O\left(\kappa^{-\frac{3}{2}}\right)$

$$
\begin{aligned}
F_{k}(z) & =\frac{k !}{\sqrt{ } \pi \Gamma(\kappa+1) z}\left\{1+\frac{z^{2}-1}{2 \kappa z^{2}}+O\left(k^{-2}\right)\right\} \\
& =\frac{1}{z \sqrt{\pi k}}\left\{1+\frac{1}{2 k}\left(\frac{1}{4}-\frac{1}{z^{2}}\right)+O\left(k^{-2}\right)\right\}, \quad k \rightarrow \infty
\end{aligned}
$$

when $|z|>0$ and $|\arg (z)| \leq \frac{1}{2} \pi$, where in (C.8) we have employed the expansion of the ratio of gamma functions $[1$, p. 257].

The behavior of $F_{k}(z)$ in the sectors $\frac{1}{2} \pi<|\arg (z)| \leq \pi$ for $|z|$ bounded away from zero can be obtained from (C.4) and (C.8) as

$$
F_{k}(z)=2\left(1-z^{2}\right)^{-\kappa}+\frac{1}{z \sqrt{\pi k}}\left\{1+\frac{1}{2 k}\left(\frac{1}{4}-\frac{1}{z^{2}}\right)+O\left(k^{-2}\right)\right\}, \quad k \rightarrow \infty .
$$

\footnotetext{
${ }^{8} \mathrm{It}$ is routine to verify, by consideration of the mapping $\chi=z^{2} /\left(1-z^{2}\right)$, that $\left|\arg \left(\chi^{1 / 2}\right)\right| \leq \pi / 2$ when $|\arg (z)| \leq \pi / 2$. We omit these details.
} 


\section{References}

1. M. Abramowitz and I. Stegun, (Eds.) Handbook of Mathematical Functions, New York, Dover, 1965.

2. M. V. Berry and C. J. Howls, Unfolding the high orders of asymptotic expansions of integrals with coalescing saddles: singularity theory, crossover and duality, Proc. Roy. Soc. Lond. A443 (1993), 107-126.

3. W. G. C. Boyd, Gamma function asymptotics by an extension of the method of steepest descents, Proc. Roy. Soc. Lond. A447 (1994), 609-630.

4. O. Diekmann, Asymptotic expansion of certain numbers related to the gamma function, Report TN 80, Mathematical Centre, Amsterdam, 1975.

5. R. B. Dingle, Asymptotic Expansions: Their Derivation and Interpretation, Academic Press, London, 1973.

6. T. M. Dunster, Asymptotic solutions of second-order linear differential equations having almost coalescent turning points, with an application to the incomplete Gamma function, Proc. Roy. Soc. Lond. A452 (1996), 1331-1349.

7. _ـ Error analysis in a uniform asymptotic expansion for the generalised exponential integral, J. Comp. and App. Math. 80 (1997), 127-161.

8. F. W. J. Olver, Asymptotics and Special Functions, Academic Press, New York, 1974. Reprinted by A.K. Peters, Wellesley, 1997.

9. $ـ$ The generalized exponential integral, Int. Series Num. Math. 119 (1994), 497-509.

10. R. B. Paris, An asymptotic representation for the Riemann zeta function on the critical line, Proc. Roy. Soc. Lond. A446 (1994), 565-587.

11. R. B. Paris and S. Cang, An asymptotic representation for the Riemann zeta function, Methods and Applic. of Analysis 4 (1997), 449-470.

12. L. J. Slater, Generalised Hypergeometric Functions, Cambridge University Press, Cambridge, 1966.

13. N. M. Temme, The asymptotic expansion of the incomplete Gamma functions, SIAM J. Math. Anal. 10 (1979), 757-766.

14. _ Special Functions, Wiley, New York, 1996.

Department of Mathematical Sciences, San Diego State University, San Diego, California 92182-7720, USA

Division of Mathematical Sciences, University of Abertay Dundee, Dundee DD1 1HG, UK 